\title{
Bounds on OPE coefficients from interference effects in the conformal collider
}

\author{
Clay Córdova, ${ }^{a}$ Juan Maldacena ${ }^{a}$ and Gustavo J. Turiaci ${ }^{b}$ \\ ${ }^{a}$ School of Natural Sciences, Institute for Advanced Study, \\ 1 Einstein Drive, Princeton, NJ, U.S.A. \\ ${ }^{b}$ Physics Department, Princeton University, \\ Jadwin Hall, Washington Road, Princeton, NJ, U.S.A. \\ E-mail: claycordova@ias.edu, malda@ias.edu, turiaci@princeton.edu
}

ABSTRACT: We apply the average null energy condition to obtain upper bounds on the three-point function coefficients of stress tensors and a scalar operator, $\langle T T \mathcal{O}\rangle$, in general CFTs. We also constrain the gravitational anomaly of $\mathrm{U}(1)$ currents in four-dimensional CFTs, which are encoded in three-point functions of the form $\langle T T J\rangle$. In theories with a large $N$ AdS dual we translate these bounds into constraints on the coefficient of a higher derivative bulk term of the form $\int \phi W^{2}$. We speculate that these bounds also apply in de-Sitter. In this case our results constrain inflationary observables, such as the amplitude for chiral gravity waves that originate from higher derivative terms in the Lagrangian of the form $\phi W W^{*}$.

Keywords: AdS-CFT Correspondence, Conformal Field Theory, Anomalies in Field and String Theories

ARXIV EPRINT: 1710.03199 


\section{Contents}

1 Introduction 2

2 ANEC and the conformal collider 3

2.1 The average null energy condition 3

2.2 The conformal collider 4

2.2.1 External states created by $T \quad 5$

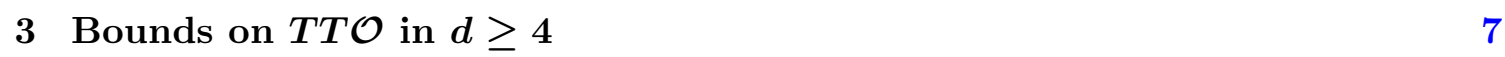

3.1 Analysis of the bound 9

$\begin{array}{lll}3.2 & \text { Free field theories and destructive interference } & 10\end{array}$

4 Bounds on $T T \mathcal{O}$ in $d=3 \quad 12$

$\begin{array}{lll}4.1 & \text { Chern-Simons matter theories } & 14\end{array}$

$\begin{array}{lll}4.2 & 3 \mathrm{~d} \text { Ising model } & 15\end{array}$

5 Bounds on $T T J$ in $d=4 \quad 16$

$\begin{array}{lll}5.1 & \text { Supersymmetry and the } R \text {-current } & 18\end{array}$

6 Bounds on coefficients of the AdS effective action 19

7 Constraints for de-Sitter and inflation $\quad 21$

7.1 Comments on scalar-tensor-tensor three-point functions 23

A Absence of positive local operators $\quad 24$

B Details of the collider calculation $\quad 25$

$\begin{array}{ll}\text { B.1 Normalized states } & 25\end{array}$

B.2 Three-point functions 26

$\begin{array}{lll}\text { B.3 Energy matrix } & 28\end{array}$

$\begin{array}{ll}\text { C Free scalar correlators } & 31\end{array}$

$\mathrm{D}\langle T T O\rangle$ parity-odd structures in $d=3 \quad 32$

$\mathrm{E}\langle T T J\rangle$ three-point function 32

F Computing the bound in the gravity theory 33

$\begin{array}{lll}\text { F.1 Four-dimensional case } & 35\end{array}$ 


\section{Introduction}

In this paper we investigate some implications of the average null energy condition in conformal field theories. We consider the conformal collider physics experiment discussed in [1]. In that setup, we produce a localized excitation by acting with a smeared operator near the origin of spacetime. Then we measure the energy flux at infinity per unit angle. Requiring that the energy flux is positive imposes constraints on the three-point function coefficients. This method was used to constrain three-point functions of the stress tensor in [1-3].

In this paper we use this same method to constrain the three-point functions of two stress tensors and another operator $\langle T T \mathcal{O}\rangle$. The new idea consists of creating the initial state by a linear combination of a stress tensor operator and the operator $\mathcal{O}$. The threepoint function $\langle T T \mathcal{O}\rangle$ appears as a kind of interference term in the expression for the energy. Requiring that the total contribution to the energy flux is positive imposes a nontrivial upper bound on the absolute magnitude of this three-point correlator. We apply these ideas to general scalar operators $\mathcal{O}$ as well as conserved currents with spin one, $J$, where we use it to put bounds on the gravitational anomaly in $d=4$ CFTs. Because the bound arises from quantum mechanical interference effects, these bounds are stronger than those obtained in states created by a single primary local operator and its descendants (though the resulting bounds involve more OPE coefficients).

This energy flux at infinity is given by an integral of the stress tensor. On the boundary of Minkowski space this integral is simply the average null energy $\mathcal{E}=\int d x^{-} T_{--}$. We review this in section 2. Physically, we expect that this energy should be positive for all angles. Recently, the average null energy condition was proven using entanglement entropy methods [4] as well as reflection positivity euclidean methods [5]. When we create a localized state using the stress tensor, this energy distribution is completely determined by the three-point function of the stress tensor. Two of the insertions correspond to the insertions creating the state in the bra and the ket. The third corresponds to the one measuring the energy flux at infinity. The resulting bounds could also be obtained by requiring standard reflection positivity of the euclidean theory [6, 7]. However, the conformal collider calculations provide an efficient way to extract the results.

One of our main results is a sum rule constraining the OPE coefficients of scalar primary operators $\mathcal{O}$ with the energy-momentum tensor $T$. In spacetime dimensions $d \geq 4$ there is a single OPE coefficient controlling the $\langle T T \mathcal{O}\rangle$ three-point function. We find that this data is constrained as

$$
\sum_{\text {Scalar Primaries }}\left|C_{T T \mathcal{O}_{i}}\right|^{2} f\left(\Delta_{i}\right) \leq N_{B}
$$

where $N_{B}$ is one of the three OPE coefficients in $\langle T T T\rangle$ (the one occurring in a theory of free bosons), and the non-negative function $f(\Delta)$ is given explicitly by

$$
f(\Delta)=\frac{(d-1)^{3} d \pi^{2 d} \Gamma\left(\frac{d}{2}\right) \Gamma(d+1) \Gamma(\Delta) \Gamma\left(\Delta-\frac{d-2}{2}\right)}{(d-2)^{2} \Gamma\left(\frac{\Delta}{2}+2\right)^{4} \Gamma\left(\frac{d+\Delta}{2}\right)^{2} \Gamma\left(d-\frac{\Delta}{2}\right)^{2}} .
$$

This function arises by doing the integrals involved in smearing the operator as well as in computing the energy flux. We derive this bound in detail in section 3 , and discuss 
some simple physical consequences such as its interpretation in free field theories, large $N$ holographic systems, and general implications for the asymptotics of OPE coefficients.

In section 4 we consider analogous results in spacetime dimension three. This case is special because the three-point functions of interest admit both parity preserving and parity violating structures. The bounds we find generalize those recently obtained in [8]. We apply our results to large $N$ Chern-Simons matter theories, and further use them to obtain predictions on OPE coefficients $C_{T T \mathcal{O}}$ for scalars in the Ising model using the recent results of the conformal bootstrap [9]. For instance, we find that operator $\varepsilon$ has an OPE coefficient constrained as

$$
\left|C_{T T \varepsilon}\right| \leq 1.751\left|C_{T T: \phi^{2}:}\right|
$$

where the right-hand side is the value in the free scalar theory based on the field $\phi$.

In section 5 we consider bounds in four-dimensional CFTs with a global symmetry current $J$. We apply the same techniques to obtain universal constraints on the gravitational anomaly of the current $J$.

In section 6 we show that the $\langle T T \mathcal{O}\rangle$ correlator can be generated from a gravity theory in $A d S_{d+1}$ through a higher derivative term, $\int \phi W^{2}$, in the bulk effective action. We match the coefficient of this term to the $C_{T T \mathcal{O}}$ coefficient in the boundary theory by performing the same collider experiment in the bulk, where it involves propagation through a shock wave. One interesting feature of this presentation is that the resulting bound is independent of the mass of $\phi$. Thus, the $\Delta$ dependence of (1.1) is purely kinematic and results from translating the boundary three-point function coefficient to a bulk interaction. We use our AdS presentation to show that $\alpha^{\prime}$ corrections satisfy the bound.

In section 7 we extrapolate the bounds we obtained in AdS to "quasi bounds" on the coefficients of the effective action in de Sitter space. We call them "quasi-bounds" because, unfortunately, for de-Sitter we do not know how to prove a sharp bound. We can think of these as a good indication for where the bulk effective theory should break down. We apply these "quasi-bounds" to constrain the amplitude of chiral gravity waves, and to constrain the violations of the inflationary "consistency condition" for the two-point function. Both of these effect arise from higher curvature couplings of the form $\phi W^{2}$ or $\phi W W^{*}$.

In the appendices we include more explicit derivations of the material in the main sections.

\section{ANEC and the conformal collider}

\subsection{The average null energy condition}

The null energy condition is a central assumption in many classical theorems of general relativity. These results allow us to exclude unphysical spacetimes where causality violation, naked singularities, or other physical pathologies occur [10].

If we move beyond classical field theory, these results appear to be in doubt. Quantum effects lead to fluctuations that prohibit any local operator from having a positive expectation value in every state [11]. (We review these ideas in appendix A.) In particular the 
(a)

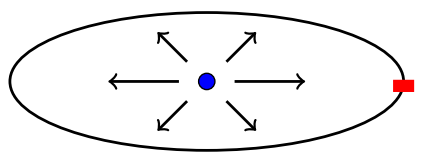

(b)

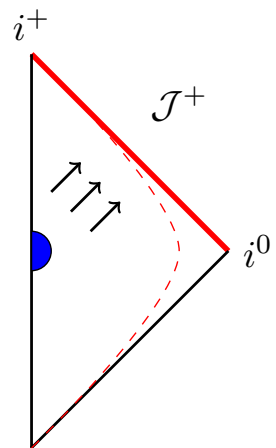

Figure 1. In the conformal collider experiment (a), the energy created by a localized excitation (blue) is measured far away by a calorimeter (red). (b) For a CFT, this is equivalent to measuring the energy at null infinity $\mathcal{J}^{+}$.

local energy density and other components of the energy-momentum tensor have negative expectation value in some states.

Deeper investigation reveals a potential resolution. While components of the energymomentum tensor are pointwise non-positive, a weaker hypothesis, the so-called average null energy condition, is often sufficient to enforce causal behavior [12]. This condition states that the integral along a complete null geodesic of the null energy density is a positive definite operator

$$
\mathcal{E}=\int_{-\infty}^{\infty} d x^{-} T_{--} \geq 0
$$

Recently there has been significant interest in understanding the average null energy condition (2.1) in the context of local quantum field theories. In [5], an argument was given establishing (2.1) in conformal field theories by examining the constraints of causality on the light-cone operator product expansion. In [4], an alternative argument was given linking the average null energy operator to entanglement entropy, then establishing positivity using strong subadditivity. These information theoretic methods have also been extended to obtain new inequalities strengthening (2.1) [13].

Given that the average null energy in quantum field theory is now a theorem, it is interesting to take it as input and use it to constrain conformal field theory data.

\subsection{The conformal collider}

An efficient way to extract consequences of the average null energy condition in CFTs is to use the conformal collider setup of [1]. This technique is closely related to deep inelastic scattering experiments in conformal field theory $[1,14]$. As we review, in the context of AdS/CFT these bounds arise from demanding causality of the bulk theory in a shockwave background.

The specific physical problem of interest is to create a disturbance in a conformal field theory and then to measure the correlation of energy deposited at various angles at future null infinity (see figure 1). 
The states in which we measure the energy are obtained by acting with local operators $\mathcal{O}(x)$ on the Lorentzian vacuum $|0\rangle$. We further give these states definite timelike momentum $q .{ }^{1}$ Thus we examine the state

$$
|\mathcal{O}(q, \lambda)\rangle=\mathcal{N} \int d^{d} x e^{-i q t} \lambda \cdot \mathcal{O}(x)|0\rangle,
$$

where $\lambda$ is a polarization tensor accounting for the possible spin of $\mathcal{O}$, and $\mathcal{N}$ is a normalization factor defined such that (2.2) has unit norm.

We now measure the energy at null infinity in this state. In $d$ dimensions null infinity is a sphere $S^{d-2}$ and we parameterize it by a unit vector $n$.

$$
\langle\mathcal{E}(n)\rangle_{\lambda \cdot \mathcal{O}}=\lim _{r \rightarrow \infty} r^{d-2} \int_{-\infty}^{\infty} d x^{-}\left\langle\mathcal{O}(q, \lambda)\left|T_{--}\left(x^{-}, r n\right)\right| \mathcal{O}(q, \lambda)\right\rangle .
$$

The average null energy condition implies that the resulting function is non-negative as a function of the direction $n$.

Since we are working in a conformal field theory this energy expectation value may be explicitly evaluated. Indeed the object being integrated in (2.3) is a three-point function $\langle\mathcal{O} T \mathcal{O}\rangle$ in Lorentzian signature with a prescribed operator ordering. Thus, the result of (2.3) is an explicit function of OPE coefficients.

\subsubsection{External states created by $T$}

Let us review the essential details of this calculation in the case where the external state is created by an energy momentum tensor. In general in $d \geq 4$ spacetime dimensions, the three-point function of energy-momentum tensors may be parameterized in terms of three independent coefficients

$$
\langle T T T\rangle=N_{B}\langle T T T\rangle_{B}+N_{F}\langle T T T\rangle_{F}+N_{V}\langle T T T\rangle_{V}
$$

where the various $B, F, V$ structures are those that arise in a theory of respectively free bosons, fermions, or $(d-2) / 2$ forms. ${ }^{2}$ Our conventions are such that for free fields, $N_{B}$ counts the number of real scalars, $N_{F}$ the total number of fermionic degrees of freedom (e.g. it is $2^{\lfloor d / 2\rfloor}$ for a Dirac fermion), and $N_{V}$ counts the number of degrees of freedom in a $(d-2) / 2$ form (for a single such field this number is $\left.\Gamma(d-1) / \Gamma(d / 2)^{2}\right)$.

A single linear combination of these coefficients is fixed by the conformal Ward identity, and related to the two-point function coefficient $C_{T}$ of energy momentum tensors (see equation (B.7) for our conventions on the two-point function)

$$
C_{T}=\frac{1}{\Omega_{d-1}^{2}}\left(\frac{d}{d-1} N_{B}+\frac{d}{2} N_{F}+\frac{d^{2}}{2} N_{V}\right)
$$

\footnotetext{
${ }^{1}$ For technical reasons it is sometimes useful to create a localized wavepacket instead of an exact momentum eigenstate. This subtlety will not affect our discussion.

${ }^{2}$ In odd $d$ there is no free field associated to the structure parameterized by $N_{V}$, but nevertheless there is still a structure. See $[3,15]$ for details.
} 
where $\Omega_{n}$ is the area of a sphere $S^{n}{ }^{3}$ As another point of reference let us briefly specialize to the case of four-dimensional theories. In that case, the coefficients of the three-point function are related to conformal anomalies $a, c$ that parameterize the trace of the energymomentum tensor in a general metric background

$$
\left\langle T_{\mu}^{\mu}\right\rangle[g]=\frac{c}{16 \pi^{2}} W^{2}-\frac{a}{16 \pi^{2}} E^{2},
$$

where $W$ is the Weyl tensor and $E$ is the Euler density. The coefficient $c$ is proportional to $C_{T}$, while

$$
a=\frac{1}{1440}\left(4 N_{B}+11 N_{F}+124 N_{V}\right) .
$$

Returning to case of general dimensions we now investigate the null energy operator using these three-point functions. It is useful to organize the calculation using the relevant symmetries, which are rotations on the null $S^{d-2}$. In addition, the three-point function of $T$ 's is parity invariant. ${ }^{4}$ It follows that the most general expression for the null energy is

$$
\langle\mathcal{E}(n)\rangle_{\lambda \cdot T}=\frac{q}{\Omega_{d-2}}\left[1+t_{2}\left(\frac{\lambda_{i j}^{*} \lambda_{i k} n^{j} n^{k}}{|\lambda|^{2}}-\frac{1}{d-1}\right)+t_{4}\left(\frac{\lambda_{i j}^{*} \lambda_{k l} n^{i} n^{j} n^{k} n^{l}}{|\lambda|^{2}}-\frac{2}{d^{2}-1}\right)\right],
$$

where the constants have been fixed so that the total energy of the state is $q$, and $t_{2}$ and $t_{4}$ are computable functions of $N_{B}, N_{F}, N_{V}$.

A useful way to understand the answer is to view the vector $n$ as fixed and to decompose the states (parameterized by their polarizations) under the remaining symmetry group $\mathrm{SO}(d-2)$. For example, the polarization that has spin zero under rotations around the $\vec{n}$ axis is

$$
\lambda_{i j}^{0} \propto\left(n_{i} n_{j}-\frac{\delta_{i j}}{(d-1)}\right)
$$

In a similar way we can write polarization tensors that have spin one and spin two under rotation around the $\vec{n}$ axis. The energy flux in the direction $n$ is the same for every state in a fixed $\mathrm{SO}(d-2)$ representation, and we denote them by $q T_{i} / \Omega_{d-2}$. Explicitly carrying out the integrals gives:

$$
\begin{aligned}
& T_{0}=\left(1-\frac{t_{2}}{d-1}-\frac{2 t_{4}}{d^{2}-1}\right)+\frac{d-2}{d-1}\left(t_{2}+t_{4}\right)=\rho_{0}(d)\left(\frac{N_{B}}{C_{T}}\right) \\
& T_{1}=\left(1-\frac{t_{2}}{d-1}-\frac{2 t_{4}}{d^{2}-1}\right)+\frac{t_{2}}{2}=\rho_{1}(d)\left(\frac{N_{F}}{C_{T}}\right) \\
& T_{2}=1-\frac{t_{2}}{d-1}-\frac{2 t_{4}}{d^{2}-1}=\rho_{2}(d)\left(\frac{N_{V}}{C_{T}}\right)
\end{aligned}
$$

where the index labels the $\mathrm{SO}(d-2)$ charge and in the above $\rho_{i}(d)$ is a positive function that depends only on the spacetime dimension (and not the OPE coefficients). Their explicit form is given in equation (B.33).

\footnotetext{
${ }^{3} \Omega_{n-1}=2 \pi^{n / 2} / \Gamma(n / 2)$.

${ }^{4}$ In $d=3$ the three-point function has a parity odd piece which we discuss in section 4 .
} 
Additional symmetries imply constraints on the parameters above. In any superconformal field theory we have $t_{4}=0$. For holographic CFTs dual to Einstein gravity the parameters are $t_{2}=t_{4}=0$, giving angle independent energy one-point functions $T_{0}=T_{1}=T_{2}=1$.

Returning to the general discussion, we can see from (2.10) that the average null energy condition implies the inequalities

$$
N_{B} \geq 0, \quad N_{F} \geq 0, \quad N_{V} \geq 0
$$

One significant remark concerning the bounds (2.11) is that they may clearly be saturated in free field theories. Conversely, it has been argued [16] that any theory that saturates the conformal collider bounds must be free. The fact that the bounds may be saturated in actual CFTs illustrates that the conformal collider is an efficient way of extracting the implications of the average null energy condition. Namely, we could not possibly get a stronger bound, otherwise we would run into a contradiction with free theories.

\section{Bounds on $T T \mathcal{O}$ in $d \geq 4$}

We now turn to our main generalization of the conformal collider bounds reviewed in section 2.2. We explore the consequences of the average null energy condition in more general states than those created by a single primary operator. Specifically in this section we will investigate states which are obtained by a linear combination of primary operators. We will find that the average null energy condition in such states yields new inequalities on OPE coefficients.

In this section, the states we consider will be created by a linear combination of the energy-momentum tensor and a general scalar hermitian operator $\mathcal{O}$. We parameterize such a state in terms of normalized coefficients $v_{i}$

$$
|\Psi\rangle=v_{1}|T(q, \lambda)\rangle+v_{2}|\mathcal{O}(q)\rangle
$$

The energy one-point function in the collider experiment is now a matrix

$$
\langle\Psi|\mathcal{E}(n)| \Psi\rangle=v^{\dagger}\left(\begin{array}{cc}
\langle T(q, \lambda)|\mathcal{E}(n)| T(q, \lambda)\rangle & \langle T(q, \lambda)|\mathcal{E}(n)| \mathcal{O}(q)\rangle \\
\langle T(q, \lambda)|\mathcal{E}(n)| \mathcal{O}(q)\rangle^{*} & \langle\mathcal{O}(q)|\mathcal{E}(n)| \mathcal{O}(q)\rangle
\end{array}\right) v
$$

The average null energy condition implies that this matrix is positive definite. This is a stronger condition than requiring that the diagonal entries are positive and will imply new inequalities on OPE coefficients.

The majority of the entries in this matrix have already been computed. For instance, in section 2.2.1 we reviewed the portion of the matrix involving the energy expectation value in states created by the energy momentum tensor. Even simpler is the entry involving the expectation value in the scalar state which gives rise to a uniform energy distribution

$$
\langle\mathcal{O}(q)|\mathcal{E}(n)| \mathcal{O}(q)\rangle=\frac{q}{\Omega_{d-2}} .
$$


It remains to determine the off-diagonal entries in the matrix. It is again useful to organize the expected answer using the rotation group on the null sphere. Clearly we have

$$
\langle T(q, \lambda)|\mathcal{E}(n)| \mathcal{O}(q)\rangle \sim \lambda_{i j} n^{i} n^{j} .
$$

Therefore, the only polarization of the energy momentum tensor that participates in the non-trivial interference terms is the scalar $T_{0}$ aligned along the axis $n$ (see equation (2.9)).

To extract this matrix element we require the three-point function $\langle T T \mathcal{O}\rangle$. In all $d \geq 4$, the conservation constraints on $T$ imply that this correlator is fixed in terms of a single OPE coefficient $C_{T T \mathcal{O}}$. We set conventions for our normalization of this OPE coefficient by examining a simple OPE channel. Specifically we restrict all operators to a two-plane, spanned by complex coordinates $z, \bar{z}$. Then the OPE is

$$
T_{z z}(z) T_{\bar{z} \bar{z}}(0) \sim \frac{C_{T T \mathcal{O}}}{|z|^{2 d-\Delta}} \mathcal{O}(0) .
$$

If we further assume that $\mathcal{O}$ is hermitian then the OPE coefficient $C_{T T \mathcal{O}}$ is real. Additional details of this correlator including the full $d$-dimensional Lorentz covariant OPE and relation to the spinning correlator formalism of [17] are given in appendix B.

Based on these remarks, we can in general parameterize the energy flux in the direction $n$ coming from the off-diagonal matrix element (3.4) as

$$
\left\langle T\left(q, \lambda_{0}\right)|\mathcal{E}(n)| \mathcal{O}(q)\right\rangle=\frac{q}{\Omega_{d-2}}\left(\frac{C_{T T \mathcal{O}}}{\sqrt{C_{T} C_{\mathcal{O}}}} h(\Delta)\right),
$$

where $h(\Delta)$ is some universal function that may be extracted from the conformal collider calculation, and the factors of $C_{T}$ and $C_{\mathcal{O}}$ arise from normalizing the states. The relevant portion of the energy matrix (3.2) is two-by-two and takes the form

$$
\frac{q}{\Omega_{d-2}}\left(\begin{array}{cc}
T_{0} & \frac{C_{T T \mathcal{O}}}{\sqrt{C_{T} C_{\mathcal{O}}}} h(\Delta) \\
\frac{C_{T T \mathcal{O}}^{*}}{\sqrt{C_{T} C_{\mathcal{O}}}} h(\Delta) & 1
\end{array}\right) .
$$

Positivity of this matrix therefore leads to the constraint

$$
\frac{\left|C_{T T \mathcal{O}}\right|^{2}}{C_{T} C_{\mathcal{O}}}|h(\Delta)|^{2} \leq T_{0}
$$

More generally we may instead consider the collider experiment in a state created by $T$ plus a general linear combination of primary scalar operators. Positivity of the resulting energy matrix is then equivalent to the following sum rule

$$
\sum_{\text {Scalar Primaries }} \frac{\left|C_{T T \mathcal{O}_{i}}\right|^{2}}{C_{T} C_{\mathcal{O}}}\left|h\left(\Delta_{i}\right)\right|^{2} \leq T_{0} .
$$

In appendix B we explicitly compute the function $h(\Delta)$ (see equation (B.48)). By combining the result with the expression (2.10), we may reexpress the bound as

$$
\sum_{\text {Scalar Primaries }} \frac{\left|C_{T T \mathcal{O}_{i}}\right|^{2}}{C_{\mathcal{O}}} f\left(\Delta_{i}\right) \leq N_{B}
$$

where $f(\Delta)$ is given as

$$
f(\Delta)=\frac{(d-1)^{3} d \pi^{2 d} \Gamma\left(\frac{d}{2}\right) \Gamma(d+1) \Gamma(\Delta) \Gamma\left(\Delta-\frac{d-2}{2}\right)}{(d-2)^{2} \Gamma\left(\frac{\Delta}{2}+2\right)^{4} \Gamma\left(\frac{d+\Delta}{2}\right)^{2} \Gamma\left(d-\frac{\Delta}{2}\right)^{2}} .
$$




\subsection{Analysis of the bound}

We now turn to an analysis of the consequences of the general bound (3.10). The function $f(\Delta)$ has a number of significant properties. ${ }^{5}$

- Expanded near the unitarity bound we find a first order pole:

$$
f\left(\frac{d-2}{2}+x\right) \sim \frac{1}{x}
$$

Therefore in any family of theories, an operator $\mathcal{O}$ which is parametrically becoming free (i.e. $\Delta=(d-2) / 2+x$ with $x$ tending to zero) must have $\left|C_{T T \mathcal{O}}\right|$ vanish at least as fast as $\sqrt{x}$.

- For large $\Delta$ we find exponential growth

$$
f(\Delta) \sim \frac{4^{\Delta}}{\Delta^{\frac{7 d}{2}+4}}
$$

We may use this growth to approximate the sum in the bound for scalar operators of large $\Delta$. Indeed, let $\rho(\Delta)$ denote the asymptotic density of scalar primary operators. From convergence of the sum we then deduce that for large $\Delta$ the spectral weighted OPE coefficients must decay exponentially fast

$$
\rho(\Delta) \frac{\left|C_{T T \mathcal{O}}\right|^{2}}{C_{\mathcal{O}}} \leq \frac{\Delta^{\frac{7 d}{2}+3}}{4^{\Delta}} .
$$

These estimates agree with those implied by convergence of the OPE expansion found in [19] for scalar operators.

- If $\Delta$ is an even integer greater than or equal to $2 d$ we find that $f(\Delta)$ vanishes. We can understand the necessity of this as follows. We can imagine a large $N$ CFT dual to weakly coupled theory of gravity. In such theories we can consider the sequence of operators $\mathcal{O}=: T^{A B} \partial^{2 n} T_{A B}:$ At large $N$ the dimensions of these operators are fixed to $\Delta=2 d+2 n$. Moreover, for these operators $\frac{C_{T T \mathcal{O}}^{2}}{C_{\mathcal{O}}}$ is of order $C_{T}^{2}$. Thus, compatibility with the bound (3.10) for large $C_{T}$, requires that $f(\Delta)$ vanishes at these locations.

The above argument does not explain why $f(\Delta)$ has double zeros. But the double zeros imply that the bound may be obeyed at subleading order, where we include the anomalous dimensions of these operators which scale as $1 / C_{T}$, by truncating the sum on $n .^{6}$

- The function $f(\Delta)$ is non-zero for $\Delta=d$. Therefore the bound (3.10) may be applied to marginal operators. In that context, it constrains the change in $C_{T}$ at leading order in conformal perturbation theory.

\footnotetext{
${ }^{5}$ A function with similar properties was obtained in [18] in a different context.

${ }^{6}$ We thank E. Perlmutter for comments on this point.
} 


\subsection{Free field theories and destructive interference}

Let us investigate the bound further in free field theories. These examples are interesting because the bound (3.10) is saturated.

Consider first a theory of a free real boson $\phi$ in dimension $d$. There is a $\mathbb{Z}_{2}$ global symmetry under which $\phi$ is odd and the energy-momentum tensor $T$ is even. Therefore we need only consider scalars made from an even number of $\phi$ 's. Since the explicit expression for $T$ is quadratic in the free fields, the only possible scalars that may contribute to the bound are $: \phi^{2}:$ and $: \phi^{4}:$.

By a simple inspection of the Wick contractions we deduce that : $\phi^{4}$ : has vanishing $T T \mathcal{O}$ correlation function. ${ }^{7}$ Meanwhile : $\phi^{2}$ : has

$$
\frac{\left|C_{T T \mathcal{O}}\right|^{2}}{C_{\mathcal{O}}}=\frac{(d-2)^{4} \Gamma(d / 2+1)^{4}}{8 \pi^{2 d}(d-1)^{4}} .
$$

This exactly saturates the bound (3.10).

We can also consider the bound applied to free fields of different spin. In $d=4$ the theory of free fermions or free gauge bosons have vanishing $N_{B}$. Therefore the bound implies that for all scalar operators $\mathcal{O}$ either $C_{T T \mathcal{O}}$ vanishes, or $\mathcal{O}$ has dimension $2 d+2 n$ for non-negative integer $n$.

It is straightforward to directly verify this prediction. For instance consider the free vector. The gauge invariant field strength gives rise to two local operators $F_{\mu \nu}^{+}$and $F_{\mu \nu}^{-}$, which are respectively self-dual and anti-self-dual two-forms. Note that this free field theory enjoys a continuous electromagnetic duality symmetry under which $F_{\mu \nu}^{ \pm}$rotate with opposite charge. The energy-momentum tensor $T_{\mu \nu}$ is neutral under this transformation, and hence a scalar operator $\mathcal{O}$ with non-vanishing $C_{T T \mathcal{O}}$ must also be neutral. If we recall that $F_{\mu \nu}^{+} F^{-\mu \nu}$ vanishes identically, then we see that the lowest dimension neutral scalar operator is $\left(F_{\mu \nu}^{+} F^{+\mu \nu}\right)\left(F_{\alpha \beta}^{-} F^{-\alpha \beta}\right)$. Since this has dimension eight, the weight function $f(\Delta)$ vanishes. Moreover all other scalar operators that are neutral have larger even integer dimension. Thus, the bound is obeyed.

A more physical way to understand why the bound is saturated in the free scalar theory is to visualize the state created by local operators.

Let us consider the action of an operator with non-zero energy but zero spatial momentum. If the operator is a bilinear in the fields, such as the stress tensor in a free theory, then it will create a pair of particles with back to back spatial momenta. Of course, the operator creates a quantum mechanical superposition of states where these momenta point in various directions. For a scalar bilinear operator we get an s-wave superposition. For the stress tensor we get a superposition determined by the polarization tensor.

As in previous sections, we measure the energy in the angular direction $n$ and hence can focus on the properties of the wavefunction for the pair of particles in that particular direction. As in section 2.2.1 it is convenient to decompose the polarization tensors of the operators according to their angular momentum around the $n$ axis. We can then easily

\footnotetext{
${ }^{7}$ The contractions imply that $\left\langle T T: \phi^{4}:\right\rangle \propto\left\langle T: \phi^{2}:\right\rangle\left\langle T: \phi^{2}:\right\rangle$, which is zero since two-point functions of different operators vanish.
} 


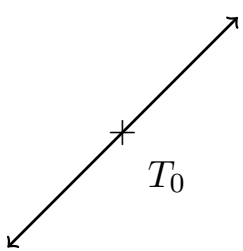

(a)

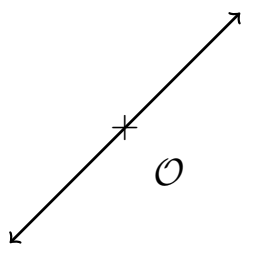

(d)

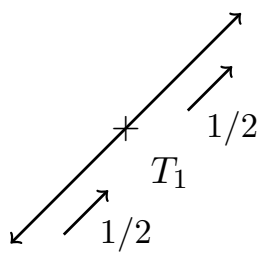

(b)

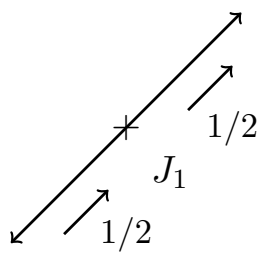

(e)

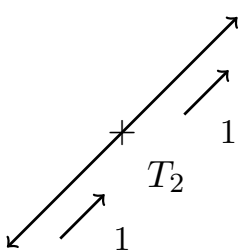

(c)

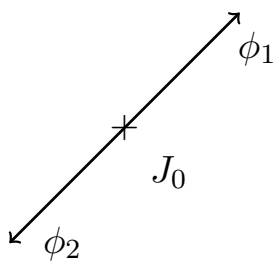

(f)

Figure 2. We consider operators with zero spatial momentum that create a pair of free particles. In $(\mathrm{a}, \mathrm{b}, \mathrm{c})$ we consider a stress tensor operator. We examine the wavefunction along the direction specified by the long arrow and we decompose the stress tensor according to the spin around that axis. (a) The spin zero state is obtained for scalars, spin one for fermions (b) and spin two for vectors or self-dual forms (c). (d) is the state produced by a scalar operator with can interfere with (a). (e) is produced by a current with spin one along the observation axis and can interfere with (b). Finally (f) is a current with spin zero along the observation axis in a theory of scalars. It produces two different real scalars in the back to back configuration and cannot interfere with (a).

check that a spin zero state $T_{0}$ can be produced only in a theory of scalars, a spin one state $T_{1}$ can be produced only in a theory of fermions, and $T_{2}$ only in a theory of vectors (or $d / 2-1$ forms), see figures $2(\mathrm{a}, \mathrm{b}, \mathrm{c})$. This explains formula 2.10.

A scalar operator of the form $\mathcal{O}=: \phi^{2}$ :, where $\phi$ is an elementary scalar, can also produce a back to back combination of scalar particles, see figure 2(d). Along the direction of observation this combination has the same form as the one produced by $T_{0}$, in figure 2 (a). It is clear that we can make a quantum mechanical superposition so that the wavefunction for the pair vanishes along that particular observation direction. This saturates the bound because we get zero energy along that direction. For that superposition of $T$ and $\mathcal{O}$ the energy along other directions is still non-zero.

A similar argument helps us understand why we also saturate the $\langle T T J\rangle$ correlator bound in the four dimensional theory of a Weyl fermion (see section 5). In that case we can make a superposition of the state $T_{1}$ in figure $2(\mathrm{~b})$ with the state $J_{1}$ in $2(\mathrm{e})$. Notice that we are using that $J$ couples to a chiral fermion. If there was another fermion with the same helicity but opposite charge, as it would be the case for a vector-like current, then we would have an additional contribution to the state created by the current that will have a relative minus sign compared to the other charged particle pair. On the other hand, for the state created by the stress tensor these two contributions have the same sign, therefore we cannot destructively interfere them. 
This highlights that the bound comes from a quantum mechanical interference effect. We saturate the bound through a destructive interference effect that prevents particles from going into a particular direction. It is important to note that this is an interference for the pair of particles. For example, if we consider a theory of scalars with a U(1) symmetry generated by a current $J$, then in a basis of real scalars the current will create two different scalars, say $\phi^{1}$ and $\phi^{2}$. This cannot interfere with the state created by the stress tensor where we have the same scalar for the two particles indicated in figure 2(a).

\section{Bounds on $T T \mathcal{O}$ in $d=3$}

In this section we will consider the case of $d=3$ separately. There are two reasons for doing this. First, the stress-tensor three-point function has two parity even structures, instead of three as in $d \geq 4$, and has a parity odd piece which is special to $d=3$. Secondly, the correlation function $\langle T T \mathcal{O}\rangle$ also has an extra parity odd structure special to $d=3$ [20].

First we consider external states created by the stress-tensor. We parametrize the three-point function of energy-momentum tensors as

$$
\langle T T T\rangle=N_{B}\langle T T T\rangle_{B}+N_{F}\langle T T T\rangle_{F}+N_{\text {odd }}\langle T T T\rangle_{\text {odd }},
$$

where $N_{B}$ and $N_{F}$ already appeared in the $d \geq 4$ case and $N_{\text {odd }}$ parametrizes a new structure. We use the same convention for the explicit expression for $\langle T T T\rangle_{\text {odd }}$ as in [8]. ${ }^{8}$ In $d \geq 4$ the energy one-point function of the collider experiment has a $\mathrm{SO}(d-2)$ symmetry for the calorimeter direction $n$. The linearly independent tensor polarizations are organized as scalar, vectors or tensors with respect to this symmetry. In $d=3$ the group becomes $\mathrm{SO}(1)$ and there are only two types of polarizations, which we take as

$$
\lambda_{0}=\frac{1}{\sqrt{2}}\left(\begin{array}{cc}
1 & 0 \\
0 & -1
\end{array}\right), \quad \lambda_{1}=\frac{1}{\sqrt{2}}\left(\begin{array}{ll}
0 & 1 \\
1 & 0
\end{array}\right) .
$$

The collider energy one-point function for an arbitrary polarization has the structure

$\langle\mathcal{E}(n)\rangle_{\lambda \cdot T}=\frac{q}{2 \pi}\left[1+t_{4}\left(\frac{\left|\lambda_{i j} n^{i} n^{j}\right|^{2}}{|\lambda|^{2}}-\frac{1}{4}\right)+d_{4} \frac{\varepsilon^{i j}\left(n_{i} n^{m} \lambda_{j m} \lambda_{k p}^{*} n^{k} n^{p}+n_{i} n^{m} \lambda_{j m}^{*} \lambda_{k p} n^{k} n^{p}\right)}{2|\lambda|^{2}}\right]$.

To obtain a bound on these parameters we can consider a state created by $|\Psi\rangle=$ $v_{1}\left|T\left(q, \lambda_{0}\right)\right\rangle+v_{2}\left|T\left(q, \lambda_{1}\right)\right\rangle$. The energy matrix becomes

$$
\langle\Psi|\mathcal{E}(n)| \Psi\rangle=\frac{q}{2 \pi} v^{\dagger}\left(\begin{array}{cc}
T_{0} & T_{\text {odd }} \\
T_{\text {odd }} & T_{1}
\end{array}\right) v
$$

where $T_{1}=1-t_{4} / 4, T_{0}=1+t_{4} / 4$ and $T_{\text {odd }}=d_{4} / 4$. These parameters were computed in [8] in terms of the $\langle T T T\rangle$ parameters $N_{B}, N_{F}$ and $N_{\text {odd }}$ obtaining

$$
C_{T} T_{1}=\frac{3}{16 \pi^{2}} N_{F}, \quad C_{T} T_{0}=\frac{3}{16 \pi^{2}} N_{B}, \quad C_{T} T_{\text {odd }}=\frac{3}{16 \pi^{2}} N_{\text {odd }} .
$$

\footnotetext{
${ }^{8}$ We identify our $N_{\text {odd }}$ with their $\pi^{4} p_{T} / 3$.
} 
For supersymmetric CFTs $t_{4}=0$ just as in the case $d \geq 4$. Also, CFTs dual to Einstein gravity have $t_{4}=d_{4}=0$.

The average null energy condition implies that the matrix (4.4) is positive definite. This implies $t_{4}$ and $d_{4}$ lie inside a circle $t_{4}^{2}+d_{4}^{2} \leq 4^{2}$, or equivalently $N_{B} \geq 0, N_{F} \geq 0$, and $N_{\text {odd }}^{2} \leq N_{B} N_{F}$.

Now we will generalize this construction along the same lines as presented in section 3 . We will consider a superposition between stress tensor and a scalar operator states

$$
|\Psi\rangle=v_{1}\left|T\left(q, \lambda_{0}\right)\right\rangle+v_{2}\left|T\left(q, \lambda_{1}\right)\right\rangle+v_{3}|\mathcal{O}(q)\rangle
$$

As anticipated above, for $d=3$ the correlation function $\langle T T \mathcal{O}\rangle$ is now determined by two parameters

$$
\langle T T \mathcal{O}\rangle=C_{T T O}^{\text {even }}\langle T T \mathcal{O}\rangle_{\text {even }}+C_{T T O}^{\text {odd }}\langle T T \mathcal{O}\rangle_{\text {odd }},
$$

where the even part is given by specializing the arbitrary $d$ correlator $d=3$, and our choice of normalization for the odd part is given explicitly in appendix D. We can make our conventions for this latter term as in (3.5) in the following way. We can define $C_{T T \mathcal{O}}^{\text {odd }}$ by the following OPE

$$
T_{z z}(z, \bar{z}, y=0) T_{z y}(0) \sim C_{T T \mathcal{O}}^{\text {odd }} \frac{\bar{z}}{4|z|} \mathcal{O}(0)
$$

where the three spatial coordinates are $(z, \bar{z}, y)$.

Using this normalization, the energy one-point function is given in terms of a threeby-three matrix as

$$
\langle\Psi|\mathcal{E}(n)| \Psi\rangle=\frac{q}{2 \pi} v^{\dagger}\left(\begin{array}{ccc}
T_{0} & T_{\text {odd }} & \frac{C_{T T O}^{\text {even }}}{\sqrt{C_{T} C_{\mathcal{O}}}} h_{3 d}^{\text {even }}(\Delta) \\
T_{\text {odd }} & T_{1} & \frac{C_{T T O}^{\text {odT }}}{\sqrt{C_{T} C_{\mathcal{O}}}} h_{3 d}^{\text {odd }}(\Delta) \\
\frac{C_{T T O}^{\text {even }}}{\sqrt{C_{T} C_{\mathcal{O}}}} h_{3 d}^{\text {even }}(\Delta) & \frac{C_{T T O}^{\text {odd }}}{\sqrt{C_{T} C_{\mathcal{O}}}} h_{3 d}^{\text {odd }}(\Delta) & 1
\end{array}\right) v
$$

where the functions $h_{3 d}^{\text {even }}(\Delta)$ and $h_{3 d}^{\text {odd }}(\Delta)$ can be obtained repeating the procedure reviewed in appendix $\mathrm{B}$ and we obtain

$$
\begin{aligned}
h_{3 d}^{\text {odd }}(\Delta) & =\frac{12 \sqrt{6} \pi^{2} \sqrt{\Gamma(2 \Delta-1)}}{\Gamma\left(\frac{\Delta+1}{2}\right) \Gamma(\Delta+3)} \frac{1}{\Gamma\left(\frac{7-\Delta}{2}\right)}, \\
h_{3 d}^{\text {even }}(\Delta) & =\frac{12 \sqrt{6} \pi^{2} \sqrt{\Gamma(2 \Delta-1)}}{\Gamma\left(2+\frac{\Delta}{2}\right) \Gamma(\Delta+3)} \frac{1}{\Gamma\left(3-\frac{\Delta}{2}\right)} .
\end{aligned}
$$

Demanding positive definiteness of the energy matrix gives several types of constraints which involve the scalar OPE coefficients. Two of these bounds are easy to generalize to an arbitrary number of scalar operators

$$
\sum_{i} \frac{\left|C_{T T \mathcal{O}_{i}}^{\text {even }}\right|^{2}}{C_{\mathcal{O}_{i}}} f_{\text {even }}\left(\Delta_{i}\right) \leq N_{B}, \quad \sum_{i} \frac{\left|C_{T T \mathcal{O}_{i}}^{\text {odd }}\right|^{2}}{C_{\mathcal{O}_{i}}} f_{\text {odd }}\left(\Delta_{i}\right) \leq N_{F}
$$


where we defined $f_{\text {odd/even }}=16 \pi^{2}\left|h_{3 d}^{\text {odd/even }}\right|^{2} / 3$. We can consider the positivity of the determinant of the $3 \times 3$ matrix. This gives an independent bound which together with the bound on $\langle T T T\rangle$ is sufficient for the positivity of the energy one-point function

$$
\begin{aligned}
& N_{B} \frac{\left|C_{T T \mathcal{O}_{i}}^{\text {even }}\right|^{2} f_{\text {even }}\left(\Delta_{i}\right)}{C_{T} C_{\mathcal{O}_{i}}}+N_{F} \frac{\left|C_{T T \mathcal{O}_{i}}^{\text {odd }}\right|^{2} f_{\text {odd }}\left(\Delta_{i}\right)}{C_{T} C_{\mathcal{O}_{i}}} \\
& -2 N_{\text {odd }} \frac{\operatorname{Re}\left[C_{T T \mathcal{O}_{i}}^{\text {even }} \sqrt{f_{\text {even }}\left(\Delta_{i}\right)} C_{T T \mathcal{O}_{i}}^{\text {odd }} \sqrt{f_{\text {odd }}\left(\Delta_{i}\right)}\right]}{C_{T} C_{\mathcal{O}_{i}}} \leq N_{B} N_{F}-N_{\text {odd }}^{2}
\end{aligned}
$$

This bound can also be generalized to include an arbitrary number of scalar operators. However, as opposed to the situation in section 3, the bounds involving different number of operators are independent. Their expressions in this case become more cumbersome and we will omit them here.

The (4.10) (4.11) have similar properties as the one appearing for the $d \geq 4$ bound. Namely they diverge at the unitarity bound $\Delta=1 / 2$ and have zeros at $6+2 n$ (even) and $7+2 n$ (odd) for integer $n$. The zeros in the even case were explained by the existence of operators with two stress tensors in theories that are dual to weakly coupled gravity, see the last point in section 3.1. The odd ones have the same explanation, except that now the scalar operators have the structure $\epsilon^{A B C} T_{A D} \partial^{2 n} \partial_{C} T_{B D}$.

\subsection{Chern-Simons matter theories}

In this section we apply the bounds derived to large $N$ Chern Simons theories at level $k$ coupled to fundamental matter. For definiteness we will consider fundamental fermions. We will denote the 't Hooft coupling by $\theta=\pi N / 2 k$. The elements of the energy matrix involving the stress tensor were computed in [8] using the explicit large $N$ expressions for the stress tensor three-point function [21]. The result is

$$
T_{1}=2 \cos ^{2} \theta, \quad T_{0}=2 \sin ^{2} \theta, \quad T_{\text {odd }}=2 \sin \theta \cos \theta .
$$

Using the conventions in, for example, [22] we can compute the off-diagonal elements involving stress-tensor mixed with a scalar operator. In the fermionic theory we consider the scalar denoted by $\mathcal{O} \sim \psi \bar{\psi}$ has dimension $\Delta=2$. The final result for the energy matrix is

$$
\langle\Psi|\mathcal{E}(n)| \Psi\rangle=\frac{q}{2 \pi} v^{\dagger}\left(\begin{array}{ccc}
2 \cos ^{2} \theta & 2 \sin \theta \cos \theta & \sqrt{2} \cos \theta \\
2 \sin \theta \cos \theta & 2 \sin ^{2} \theta & \sqrt{2} \sin \theta \\
\sqrt{2} \cos \theta & \sqrt{2} \sin \theta & 1
\end{array}\right) v .
$$

As a function of the 't Hooft coupling, this matrix has the property that all the minors have vanishing determinant. This implies saturation for all types of superposition of states. For the case of the stress tensor this was noted in [8], but we find that this is a more general feature for states where we also act with $\mathcal{O}$.

Even though we do not have a concrete physical picture explaining this, we expect a picture along the lines of section 3.2, where the interaction with the Chern-Simons gauge field has the effect of replacing free bosons or fermions by "free anyons". 


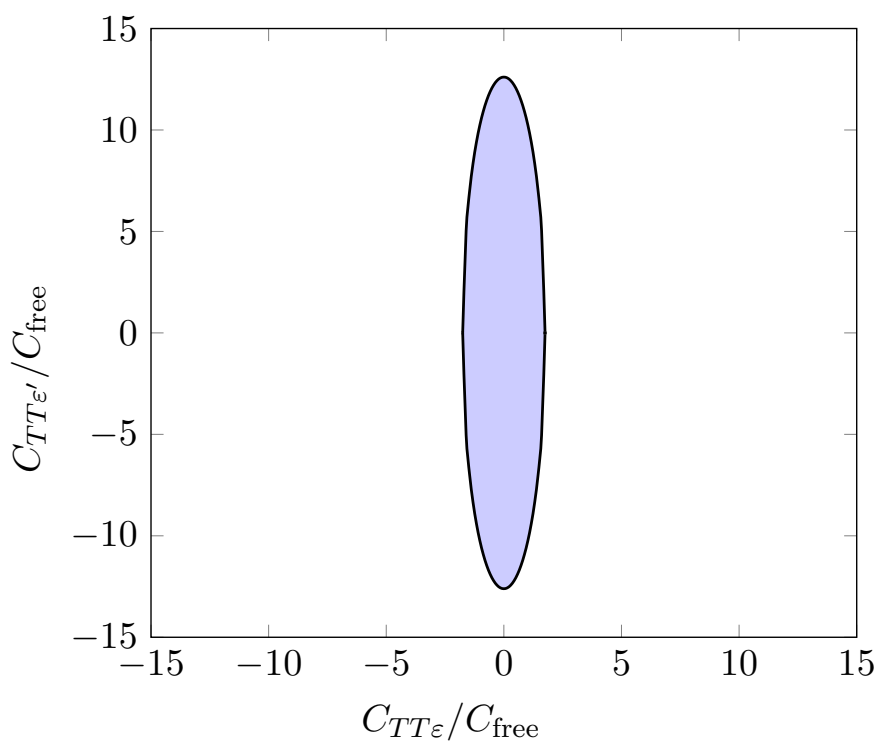

Figure 3. 3d Ising model allowed region for $C_{T T \varepsilon}$ and $C_{T T \varepsilon^{\prime}}$.

This discussion can also be applied to the case of CS coupled to fundamental bosons. From [21] we know that the energy matrix, given in terms of CFT three-point functions, can be obtained from the fermionic theory by the replacement $\theta \rightarrow \theta+\frac{\pi}{2}$ when we consider the operator $\mathcal{O} \sim \phi^{2}$ of dimension $\Delta=1$. More generally we can consider the answer (4.15) as giving the energy matrix of a large $N$ theory with a slightly broken higher spin symmetry parametrized by $\theta$.

\section{$4.2 \quad 3 d$ Ising model}

As another example, we can apply our bounds to obtain predictions for three-point coefficients for the $3 d$ Ising model. First let us parameterize the three-point coefficients of the energy-momentum tensor. Since this theory is parity preserving the coefficient $N_{\text {odd }}$ in (4.1) is necessarily zero. The remaining two structures in $\langle T T T\rangle$ have recently been computed numerically using the conformal bootstrap in $[9,23]$. Explicitly ${ }^{9}$

$$
N_{B} \approx .9334, \quad N_{F} \approx .0131
$$

The Ising model has a $\mathbb{Z}_{2}$ global symmetry under which $T$ is even. Therefore only $\mathbb{Z}_{2}$ even scalars participate in the bound. The lightest $\mathbb{Z}_{2}$ even and parity even scalar is the operator $\varepsilon$ whose dimension is known

$$
\Delta_{\varepsilon} \approx 1.4127
$$

Therefore, in a normalization where the two-point function coefficient of $\varepsilon$ is one, we can evaluate (4.12) and find the bound

$$
\left|C_{T T \varepsilon}\right| \leq .0088=(1.751) C_{\text {free }},
$$

\footnotetext{
${ }^{9}$ In making these estimates we use a value of $\theta \approx .014$. This is the central value of the calculation of [9] based on expectations for the parity odd scalar gap.
} 
where in the last equation we normalized the answer by the expression (3.15) for the value of the OPE coefficient in the free theory $C_{\text {free }}=\left|C_{T T: \phi^{2}:}\right| / \sqrt{C_{: \phi^{2}}:}$. Note that although $: \phi^{2}$ : saturates the bound in the free field theory, the dimension of $\varepsilon$ is larger than that of $: \phi^{2}$ : and hence the OPE coefficient $C_{T T \varepsilon}$ may be larger than $C_{T T: \phi^{2}:}$.

We can obtain a stronger bound by including the operator $\varepsilon^{\prime}$ of dimension $\Delta_{\varepsilon^{\prime}} \approx 3.8303$ in the sum of (4.12). Using the correct values for $f_{\text {even }}(\Delta)$ for these dimensions and normalizing by the $T T: \phi^{2}$ : OPE we obtain the constraint

$$
0.3267\left|C_{T T \varepsilon}\right|^{2}+0.0063\left|C_{T T \varepsilon^{\prime}}\right|^{2} \leq C_{\text {free }}^{2}
$$

Since the operators $\varepsilon$ and $\varepsilon^{\prime}$ are hermitian their OPE coefficients are real and the bound above defines the allowed region of OPE coefficients as the interior of an ellipse shown in figure 3 .

\section{Bounds on $T T J$ in $d=4$}

As a final example, we consider states created by a linear combination of the energymomentum tensor and a conserved vector current $J$ in $d=4$ spacetime dimensions. In this case the three-point function $\langle T T J\rangle$ is controlled by a single OPE coefficient $C_{T T J}$ and is parity violating. This three-point function is presented in detail in appendix E.

One reason why this OPE coefficient is interesting is that it is equivalent to a non-trivial mixed anomaly between the flavor symmetry generated by $J$ and the Lorentz symmetry generated by $T$ [24]. In the presence of a background metric $g$, the current $J$ is not conserved but instead obeys [25-27]

$$
\left\langle\nabla^{\mu} J_{\mu}\right\rangle[g]=\frac{C_{T T J}}{768 \pi^{2}} \epsilon^{\mu \nu \rho \sigma} R_{\mu \nu \delta \gamma} R_{\rho \sigma}^{\delta \gamma}
$$

where $R_{\mu \nu \rho \sigma}$ is the Reimann tensor.

In the above, our normalization is such that the coefficient $C_{T T J}$ may be expressed as the net chirality of the charges of elementary Weyl fermions:

$$
C_{T T J}=\sum_{\text {Left Weyl } i} q_{i}-\sum_{\text {Right Weyl } j} q_{j} .
$$

In particular, for the theory of a single Weyl fermion $C_{T T J}$ is one. In an abstract CFT without a Lagrangian presentation our normalization of the OPE coefficient is defined as follows. Fix complex coordinates $(z, w)$. Then the OPE of operators restricted to the $w=0$ plane is

$$
T_{w w}(z) T_{\bar{w} \bar{w}}(0) \sim \frac{C_{T T J}}{4 \pi^{6}|z|^{6}}\left(z J^{\bar{z}}-\bar{z} J^{z}\right) .
$$

We will also need the three-point function $\langle T J J\rangle$. This correlator is controlled by two independent coefficients:

$$
\langle T J J\rangle=Q_{C B}^{2}\langle T J J\rangle_{C B}+Q_{W F}^{2}\langle T J J\rangle_{W F}
$$


Here the structures $C B$ and $W F$ are those found for the $\mathrm{U}(1)$ current in a theory of free complex bosons $(C B)$ or free Weyl fermions $(W F)$. In a free field theory, these are expressed in terms of the charges of elementary fields as (see [15])

$$
Q_{C B}^{2}=\sum_{\text {complex scalars } i} q_{i}^{2}, \quad Q_{W F}^{2}=\sum_{\text {Weyl fermions } i} q_{i}^{2} .
$$

In general, a single linear combination of these OPE coefficients is fixed by the Ward identity. We have

$$
\langle J J\rangle \propto C_{J} \equiv \frac{1}{3}\left(Q_{C B}^{2}+2 Q_{W F}^{2}\right) .
$$

The two-point function coefficient $C_{J}$ can also be interpreted as a conformal anomaly. Indeed, in the presence of a non-trivial background gauge field that couples to $J$, the energy-momentum tensor acquires an anomalous trace. In our conventions this is

$$
\left\langle T_{\mu}^{\mu}\right\rangle[A]=\frac{C_{J}}{4} F^{\alpha \beta} F_{\alpha \beta} .
$$

We can bound the anomaly coefficient $C_{T T J}$ using the same methods described in earlier sections for scalar operators. We enforce positivity of the average null energy operator $\mathcal{E}$ in the state $|\Psi\rangle$ created by a linear combination of $T$ and $J$

$$
|\Psi\rangle=\left|T\left(q, \lambda_{T}\right)\right\rangle+\left|J\left(q, \lambda_{J}\right)\right\rangle .
$$

The expectation values $\langle\mathcal{E}\rangle_{\lambda_{T} \cdot T}$ and $\langle\mathcal{E}\rangle_{\lambda_{J} \cdot J}$ have been computed in [1]. The matrix of energy expectation values in the states $|\Psi\rangle$ may again be decomposed in terms of the $\mathrm{SO}(2)$ rotation symmetry about the vector $n$. The current operator $J$ contributes states of charge $-1,0,1$. As in the review of section 2.2.1 we may express the null energy expectation value as $\left(q J_{i} / 4 \pi\right)$ where $i$ is the $\mathrm{SO}(2)$ charge. One then finds

$$
J_{ \pm 1}=\frac{Q_{W F}^{2}}{C_{J}}
$$

By repeating the collider calculation we find that the new off-diagonal matrix element is given by

$$
\left\langle T\left(q, \lambda_{T}\right)|\mathcal{E}(n)| J\left(q, \lambda_{J}\right)\right\rangle=\frac{q}{4 \pi}\left(\sqrt{\frac{5}{\pi^{4}}} \frac{C_{T T J}}{\sqrt{C_{T} C_{J}}} \varepsilon_{i j k} \lambda_{T, i m}^{*} \lambda_{J, k} n^{m} n^{j}\right) .
$$

Note that this structure is parity odd as expected. There are other allowed parity odd expressions in terms of $\lambda_{i j}$ and $n^{i}$, but they do not arise in the null-energy matrix element. An important feature of (5.10) is that only those states of $\mathrm{SO}(2)$ charge \pm 1 can mix with the energy-momentum tensor. In particular, this means that bound will only involve the coefficient $T_{1}$ defined in (2.10).

Explicitly choosing appropriate polarization tensors we then find that positivity of the null energy matrix $\mathcal{E}$ leads to a single constraint on these OPE coefficients:

$$
C_{T T J}^{2} \leq Q_{W F}^{2} N_{W F},
$$

where $N_{W F}=N_{F} / 2$ counts the effective number of Weyl Fermions in the $\langle T T T\rangle$ correlation function. This bound is saturated in the free field theory of Weyl fermions. This can be understood using the interference argument described in section 3.2. 


\subsection{Supersymmetry and the $R$-current}

As in our analysis of scalar operators, we can generalize these results to states created by multiple currents. This is particularly interesting in the case of supersymmetric theories.

In supersymmetric theories, there is always a current $J_{R}$ contained in the same supermultiplet as $T$. In particular, since it resides in a different multiplet it can be distinguished from an ordinary flavor current $J_{F}$. We would like to improve our bound on the trace anomaly of $J_{F}$ to account for the fact that the $R$-current $J_{R}$ always exists. In order to do this we consider the state created by

$$
|\Psi\rangle=v_{1}\left|T\left(q, \lambda_{T}\right)\right\rangle+v_{2}\left|J_{R}\left(q, \lambda_{J}\right)\right\rangle+v_{3}\left|J_{F}\left(q, \lambda_{J}\right)\right\rangle .
$$

The new ingredient appearing in the calculation of the energy matrix corresponding to this state involves the three-point function $\left\langle T J_{R} J_{F}\right\rangle$. Using superconformal invariance we can fix this correlator completely. Since the details are not very illuminating we will outline the procedure. The number of parity even structures, two of them, coincides with the ones appearing in $\langle T J J\rangle$, namely relaxing permutation symmetry does not add new structures [17]. Moreover, using supersymmetric Ward identities [28] one can check that no parity odd structure is allowed for $\left\langle T J_{R} J_{F}\right\rangle \cdot{ }^{10}$ Out of the two OPE coefficients characterizing $\left\langle T J_{R} J_{F}\right\rangle$, a linear combination of them is related to the two-point function $\left\langle J_{R} J_{F}\right\rangle$, which vanishes due to superconformal invariance. This leaves $\left\langle T J_{R} J_{F}\right\rangle$ fixed by a single OPE coefficient. Finally, since $J_{R}$ lies in the same multiplet as the stress tensor we can relate this number to $C_{T T F}$, the mixed anomaly generated by the flavor current.

Combining the results outlined in the previous paragraph, and the fact that there is no new structure involved in the collider calculation, it is straightforward to obtain the off-diagonal matrix element

$$
\left\langle J_{R}\left(q, \lambda_{J}\right)|\mathcal{E}| J_{F}\left(q, \lambda_{J}\right)\right\rangle=\frac{q}{4 \pi}\left(\sqrt{\frac{20}{3 \pi^{4}}} \frac{C_{T T F}}{\sqrt{C_{T} C_{F}}}\right),
$$

where we chose $n=(1,0,0)$ and $\lambda_{J}=(0,1, i)$ for definiteness.

We can express parameters related to the $R$-current in terms of $a$ and $c=C_{T} \pi^{4} / 40$. The two-point function is related to $C_{T}$ by a supersymmetry Ward identity as $C_{R}=\frac{16}{3} c$. Its mixed anomaly is also fixed by supersymmetry to $C_{T T R}=16(c-a)$. Finally the energy one-point function is given by $J_{ \pm 1}^{R}=\frac{a}{c}[1,7]$. Supersymmetry also fixes this parameter for flavor currents as $J_{ \pm 1}^{F}=1$. Taking these facts into account allows us to write down the energy matrix as a function only of $a, c, C_{T T F}$ and $C_{F}$. We obtain

$$
\langle\Psi|\mathcal{E}| \Psi\rangle=\frac{q}{4 \pi} v^{\dagger}\left(\begin{array}{ccc}
\frac{2 c-a}{c} & \sqrt{3} \frac{c-a}{c} & \frac{1}{\sqrt{2 c}} \frac{C_{T T F}}{\sqrt{C_{F}}} \\
\sqrt{3} \frac{c-a}{c} & \frac{a}{c} & \frac{1}{\sqrt{6 c}} \frac{C_{T T F}}{\sqrt{C_{F}}} \\
\frac{1}{\sqrt{2 c}} \frac{C_{T T F}}{\sqrt{C_{F}}} & \frac{1}{\sqrt{6 c}} \frac{C_{T T F}}{\sqrt{C_{F}}} & 1
\end{array}\right) v,
$$

where for definiteness we have chosen $\lambda_{J}=(0,1, i)$ and a tensor polarization with the same $\mathrm{SO}(2)$ spin.

\footnotetext{
${ }^{10}$ This is not true for a three-point function of a stress tensor and two different conserved currents $\left\langle T J_{1} J_{2}\right\rangle$ in a generic theory.
} 
Enforcing the positivity of this matrix yields several constraints. The leading two-bytwo minor involving states $\left|T\left(q, \lambda_{T}\right)\right\rangle+\left|J_{R}\left(q, \lambda_{J}\right)\right\rangle$ gives the bound

$$
\frac{1}{2} \leq \frac{a}{c} \leq \frac{3}{2}
$$

which coincides with those derived in [1]. This bound is saturated by a free chiral multiplet, $\frac{a}{c}=\frac{1}{2}$, or a free vector multiplet, $\frac{a}{c}=\frac{3}{2}$.

To constrain the gravitational anomaly coefficient we evaluate the determinant of the full three-by-three matrix (5.14). This gives the following bound on the mixed anomaly for a flavor current

$$
\left(\frac{a}{c}-\frac{1}{2}\right)\left(36 c-24 a-\frac{C_{T T F}^{2}}{C_{F}}\right) \geq 0 .
$$

For a free chiral multiplet the bound is automatically saturated, since the first term in the left hand side vanishes independently of $C_{T T F}$. Therefore we will assume that $\frac{a}{c}>\frac{1}{2}$. Then we obtain the following bound

$$
\frac{C_{T T F}^{2}}{12 C_{F}} \leq 3 c-2 a
$$

which is stronger than the one derived in the previous section, without the use of supersymmetry. Note also that this is consistent with the free vector multiplet. In that case the right-hand-side vanishes, but there are also no flavor currents.

To conclude this section, we can mention some contexts where such bound on the mixed anomaly is relevant. First of all, when we consider holographic CFT this anomaly is related to a $5 \mathrm{~d}$ Chern-Simons term of the form $\int A \wedge R \wedge R$, where $A$ is the gauge field dual to the current $J$ (we will see in the next section how our bounds translate to bounds on the gravity couplings for the case of $T T \mathcal{O})$.

Finally, in the context of hydrodynamics and transport, quantum anomalies induce a special type of transport coefficients, see [29] and, in particular, for the mixed anomaly [3032]. The coefficient bounded in this section $C_{T T J}$, is related to the mixed anomaly recently observed experimentally in Weyl semimetals [33]. In the linear response regime, the mixed anomaly produces an energy current $\vec{j}$ given by [30-32]

$$
\vec{j}=24 C_{T T J} T^{2} \vec{B}
$$

where we denote the temperature by $T$ and the system is placed in a fixed magnetic field $\vec{B}$. This allows us to translate our results into concrete bounds for transport coefficients.

\section{Bounds on coefficients of the AdS effective action}

If the $d$ dimensional boundary theory has an $A d S_{d+1}$ dual, then we would like to translate the bounds on $C_{T T \mathcal{O}}$ to bounds on the coefficients of the bulk effective action. We are imagining that the theory has a large $N$ expansion. Then, to leading order, the bulk is given by a collection of free fields propagating on the AdS metric. The simplest interactions correspond to bulk three-point interactions. These lead to three-point functions in the 
boundary theory. For the case of gravitons we have a three-point interaction coming from the Einstein Lagrangian, but it is also necessary to include higher derivative terms, of the form $W^{2}$ and $W^{3}$, in order to get the most general structures for the tensor three-point function. It is possible to match the coefficients of the new structures to the coefficients of these higher derivative terms in the Lagrangian [1,3].

Here we consider the same problem for the case of the $\langle T T \mathcal{O}\rangle$ correlator. The first observation is that in Einstein gravity this correlator is zero, since the action of any field, expanded around the minimum of its potential has an action without a linear term in the scalar field. Notice that this also implies that a massive scalar field cannot not decay into two gravitons. However, we can write the higher derivative term

$$
S=M_{p l}^{d-1} \alpha \int d^{d+1} x \sqrt{g} \chi W^{2}
$$

in the action, where we normalized the $\chi$ field to be dimensionless. ${ }^{11}$ This term enables the field $\chi$ to decay into two gravitons. In flat space there is only one structure for the on shell three-point function between a scalar and two gravitons, except in four dimensions where threre is also a parity odd one, as we discuss later. Therefore the vertex (6.1) represents the general interaction that we can have in the theory. There can be other ways to write it which give the same three-point function as (6.1). It is possible to check that (6.1) gives rise to a $\langle T T \mathcal{O}\rangle$ three-point function with the coefficient

$$
\frac{C_{T T \mathcal{O}} \sqrt{f(\Delta)}}{\sqrt{C_{T}}}=\frac{8 \sqrt{2 d}(d-1) \pi^{d / 2}}{\sqrt{d+1} \Gamma(d / 2)} \frac{\alpha}{R_{\mathrm{AdS}}^{2}} .
$$

At first sight, it seems surprising that the function $f(\Delta)$ appearing here is the same as the one that appears in the bound (3.10). This means that the $\Delta$ dependence disappears when we express the bound in terms of $\alpha$. This is easy to understand when we derive (6.2) as follows.

First we notice that integrating the stress tensor along a null line, as in the definition of the energy measurement $\mathcal{E}=\int d x^{-} T_{--}\left(x^{-}, x^{+}=0, \vec{y}=0\right)$, we produce a shock wave in the bulk that is localized at $x^{+}=0$. We can then imagine scattering a superposition of $\chi$ and a graviton through this shock wave. This leads to a time delay that is given by a matrix mixing the graviton and the scalar. An important point is that the propagation through the shock wave is given by integrating the wave equation in a small interval before and after $x^{+}=0$. Only the shock wave contributes to this short integral over $x^{+}$, but the scalar mass term does not contribute. Therefore the time delay matrix is independent of the mass of the scalar. We can determine the precise coefficient in (6.2) by doing this explicit computation for Einstein gravity plus (6.1). We then get a bound on $\alpha$ by requiring that the time delay is positive. Comparing this to the bound (3.10) we fix the coefficient to the one in (6.2). We explain this in more detail in appendix F.

This same shock wave method enables one to set even stricter bounds on $\alpha$ if one assumes that there is a gap to the higher spin particles, ${ }^{12}$ as was discussed in [34] for the

\footnotetext{
${ }^{11}$ Here $M_{p l}$ is the reduced Planck mass in $d+1$ dimensions, defined so that the Einstein term is $S=$ $\frac{M_{p l}^{d-1}}{2} \int d^{d+1} x \sqrt{g} R$. Similarly, the action of the scalar field is $S=\frac{M_{p l}^{d-1}}{2} \int\left[(\nabla \chi)^{2}-m^{2} \chi^{2}\right]$.
} 
case of the graviton higher derivative interactions. A similar analysis can be done for the $5 \mathrm{~d}$ Chern-Simons term coupling dual to the mixed anomaly [35].

In string theory, we expect that $\alpha$ is the order of $\alpha^{\prime}$, the inverse string tension. If gravity is a good approximation, $\alpha^{\prime} \ll R^{2}$, then we find that the bound on (6.2) is far from being saturated. The bound is saturated only as the string length becomes of the order of the radius of AdS. In particular, this implies that the bound is satisfied, and far from being saturated, for the Konishi operator of $\mathcal{N}=4$ super Yang Mills at strong coupling. This operator is the lightest non-protected single trace operator which has a dimension growing like $\Delta \propto \lambda^{1 / 4}$ at strong coupling, $\lambda \gg 1$.

In the four dimensional case, we can also have a parity odd correlator with a corresponding coupling. In flat space this is related to the fact that the three-point functions with ++ or -- graviton helicities are Lorentz invariant by themselves. (The -+ graviton helicities are forbidden by angular momentum conservation). We can then write the action as

$$
S=M_{p l}^{2} \int d^{4} x \sqrt{g}\left[\frac{1}{2}(R-2 \Lambda)+\frac{1}{2}\left[(\nabla \chi)^{2}-m^{2} \chi^{2}\right]+\int \alpha_{e} \chi W^{2}+\alpha_{o} \chi W W^{*}\right],
$$

where as above we have defined $\chi$ to be dimensionless. ${ }^{13}$ In this normalization $\alpha_{i}$ has dimensions of length squared. They can be related to the coefficients of the three-point function as

$$
\frac{C_{T T \mathcal{O}}^{\text {even }} h_{\text {even }}(\Delta)}{\sqrt{C_{T}}}=\frac{24}{\sqrt{2}} \frac{\alpha_{e}}{R_{A d S_{4}}^{2}}, \quad \frac{C_{T T \mathcal{O}}^{\text {odd }} h_{\text {odd }}(\Delta)}{\sqrt{C_{T}}}=\frac{24}{\sqrt{2}} \frac{\alpha_{o}}{R_{A d S_{4}}^{2}} .
$$

The bounds in this case then read

$$
\frac{\sqrt{\alpha_{e}^{2}+\alpha_{o}^{2}}}{R_{A d S_{4}}^{2}} \leq \frac{1}{12 \sqrt{2}}
$$

in the case that there are no purely gravitational corrections to Einstein gravity. Of course, if there are three-point functions that lead to corrections to Einstein gravity, then the bound is corrected to those given in section 4 .

\section{Constraints for de-Sitter and inflation}

The physics of inflation might be our very best window into very high energy physics. The standard inflationary theory starts with a scalar field coupled to the Einstein action and includes all two (or less) derivative interactions. The universe undergoes a period of expansion that is governed by a nearly de-Sitter solution, characterized by a Hubble scale $H$ that is nearly constant. The effective coupling of the gravitational sector is of order $H / M_{p l}$ which is very small, less than $10^{-5}$. However, it is possible that there are corrections to the two derivative action due to the presence of a light string scale. The value of the string tension could be fairly low $H^{2} \lesssim T$. When the string tension becomes comparable to the Hubble scale, we expect significant corrections to the two derivative action. We do not have an explicit scenario where this happens. However, a similar situation happens in AdS space

\footnotetext{
${ }^{13}$ We also define $\left(W^{*}\right)_{\mu \nu \rho \sigma}=\frac{1}{2} \epsilon_{\mu \nu \delta \gamma} W_{\rho \sigma}^{\delta \gamma}$.
} 
when we consider a gravity dual of a not so strongly coupled large $N$ theory. Therefore it is natural to question whether something similar could happen in inflation and we can look for signatures of such a low string scale. It is important to find signatures that are as model independent as possible. Specially nice signatures are those that have a non-vanishing contribution in the de-Sitter approximation. These are not strongly suppressed by slow roll factors. In addition, their form is strongly constrained by the de-Sitter isometries. An example of such contributions are the three-point functions of gravity fluctuations, where the higher derivative corrections were discussed in [36]. Another interesting case are the couplings of the form $f_{e}(\chi) W^{2}$ or $f_{0}(\chi) W W^{*}$. These two couplings are particularly interesting because their effects are visible at the two-point function level.

Let us discuss first the parity odd coupling, which leads to chiral gravity waves [37, 38]. Namely, we have different gravity wave two-point functions, $h h_{L}, h h_{R}$, for the left and right handed circularly polarization. We can define the asymmetry $A$ as

$$
A \equiv \frac{h h^{L}-h h^{R}}{h h^{L}+h h^{R}}=4 \pi \frac{\dot{f}_{o}(\chi)}{H} H^{2}= \pm 4 \pi \sqrt{2 \epsilon}\left(\frac{\partial f}{\partial \chi}\right) H^{2}, \quad \chi=\frac{\phi}{M_{p l}},
$$

where $\chi$ is defined to be dimensionless and $\phi$ is the inflaton with canonical normalization. (The \pm comes from going from $\dot{\chi}$ to $\sqrt{\epsilon}$, since the derivative of the scalar can have either sign). If we were in $A d S_{4}$ we would have a sharp bound on the coefficients via the condition (6.5), after we identify $\alpha_{o}=\frac{\partial f}{\partial \chi}$. It is reasonable to think that in the de-Sitter case too, there will be trouble is the bound is violated. Of course, we know that even near-saturation of the bound implies that the field theory approximation is breaking down.

In the de-Sitter case we do not have a sharp derivation of a bound from boundary theory reasoning. We do not have an analog of the null energy condition, discussed in section 4, for the boundary theory, since the boundary theory is purely spacelike. It would be nice to have a sharp derivation of a de-Sitter version of the bound. In de-Sitter, we can talk of a "quasi-bound", which we get by simply applying the same bound on the coefficients of the action that we had in anti-de-Sitter. This quasi-bound should be viewed simply as an educated guess, including numerical coefficients, for the validity of bulk effective theory. A near saturation of these quasi-bounds is a strong indication of a light string scale which could also have other manifestations such as indirect evidence of higher spin massive particles, etc [39]. In summary, in de-Sitter also we have a quasi-bound on the coefficients similar to $(6.5)$, with $1 / R_{\text {AdS }} \rightarrow H$

$$
\sqrt{\left(\frac{\partial f_{e}}{\partial \chi}\right)^{2}+\left(\frac{\partial f_{o}}{\partial \chi}\right)^{2}}=\sqrt{\alpha_{e}^{2}+\alpha_{o}^{2}} \leq \frac{H^{2}}{12 \sqrt{2}}
$$

This bound, then implies a quasi-bound on the asymmetry (7.1) of the form

$$
|A| \leq \frac{4 \pi}{12} \sqrt{\epsilon} .
$$

The allowed values by this quasi-bound seem to be smaller than the smallest possible measurable value from the CMB B-modes as analyzed in [40]. Conversely, this means that 
if chiral gravity waves through E-B mode correlators are measured, then we would need a higher derivative coupling with a coefficient so large that it violates (7.2).

Let us turn now to a discussion of the parity even coupling. This coupling gives rise to a violation of the consistency condition for the two-point function [41], even in the case that the speed of sound is close to one,

$$
-8 \frac{n_{t}}{r}=1+8 H^{2} \frac{H d_{t} f_{e}}{\left(d_{t} \chi\right)^{2}}=1 \pm 8 H^{2} \frac{\alpha_{e}}{\sqrt{2 \epsilon}},
$$

where we assumed that the speed of sound for the scalar is close to one. Here $n_{t}$ is the tensor spectral index and $r$ the tensor to scalar ratio conventionally defined. Then the bound we had in (7.2) translates into the following constraint on the violation of the consistency condition

$$
\left|-8 \frac{n_{t}}{r}-1\right| \leq \frac{1}{3 \sqrt{\epsilon}}
$$

\subsection{Comments on scalar-tensor-tensor three-point functions}

The $\phi W^{2}$ higher derivative coupling between the scalar and the graviton also give rise to new contributions to the scalar-tensor-tensor three-point function. This is a contribution, that is non-vanishing in the de-Sitter limit. More precisely, if we can approximate $\partial_{\chi} f_{e}(\chi(t))$ by a constant, then we get a contribution even in de-Sitter space. The standard Einstein gravity contribution, [42], is suppressed by a slow roll factor $\sqrt{\epsilon}$, if we assume that $\partial_{\chi} f$ is of order one. Of course, our bound constrains the size of this three-point function because it is constraining the size of the coefficient $\alpha_{e} \sim \partial_{\chi} f_{e}(\chi(t))$.

The three-point function for the parity odd coupling $f_{o}(\chi) W W^{*}$ was computed in [43], where it was found to be proportional to $\partial_{\chi}^{2} f$. One might have naively expected a de-Sitter invariant contribution proportional to $\alpha_{0}=\partial_{\chi} f_{0}$, when we approximate this by a constant. The explicit computation by [43] shows that there is no such contribution. This seems surprising at first sight because this parity odd coupling does indeed give a non-vanishing contribution to the three-point function in the $A d S_{4}$ case. The reason it vanishes in deSitter is that it gives a contribution to the de-Sitter wavefunction that is a pure phase, which disappears when we take the absolute value squared of the wavefunction. The same happens with the $W^{2} W^{*}$ parity violating graviton three-point coupling [44]. The correlator proportional to $\partial_{\chi}^{2} f$ found in [43] has an extra factor of $\dot{\phi}$ and is not expected to be de-Sitter invariant (though we did not check this explicitly).

It should be noted that the correction to the two-point function consistency condition (7.4) has the right form so that the consistency condition involving the soft limit of the three-point function $[42,45]$ is obeyed, though we have not explicitly checked the precise numerical coefficients. A similar remark applies in the parity odd case; the correction to the two-point function (7.1) is such that the soft limit of the three-point function in [43] obeys the consistency condition.

\section{Acknowledgments}

We thank H. Casini, S. Giombi, R. Meyer, E. Perlmutter, D. Simmons-Duffin, and D. Stanford for discussions. We also thank E. Perlmutter for comments on a draft. C.C. is 
supported by the Marvin L. Goldberger Membership at the Institute for Advanced Study, and DOE grant DE-SC0009988. J.M. is supported in part by U.S. Department of Energy grant DE-SC0009988 and the Simons Foundation grant 385600.

\section{A Absence of positive local operators}

Let us review the essential steps of [11] in a modern language. Let $\Phi$ be any Hermitian operator and $|0\rangle$ the Lorentz invariant vacuum state. We make two assumptions:

- The one-point function $\langle 0|\Phi| 0\rangle$ vanishes.

- For all states $|\psi\rangle$ in the Hilbert space, the expectation value $\langle\psi|\Phi| \psi\rangle$ is non-negative.

Under these assumptions we may prove that $\Phi$ annihilates the vacuum state, $\Phi|0\rangle=0$. Indeed, for any positive operator, the Cauchy-Schwarz inequality implies that

$$
|\langle\psi|\Phi| 0\rangle|^{2} \leq\langle\psi|\Phi| \psi\rangle\langle 0|\Phi| 0\rangle
$$

Since the right-hand side is zero by hypothesis, we conclude that $\Phi|0\rangle$ must vanish.

If we now further assume that $\Phi$ is an operator localized within a compact region $\mathcal{R}$, we can deduce that $\Phi$ must vanish. To demonstrate this, consider operators localized in a region $\mathcal{R}^{\prime}$ that is spacelike separated from $\mathcal{R}$. Let us denote by $O_{\mathcal{R}^{\prime}}$ a set (sum of products) of smeared operators in region $\mathcal{R}^{\prime}$, then we have

$$
0=\left\langle 0\left|O_{\mathcal{R}^{\prime}}^{1} O_{\mathcal{R}^{\prime}}^{2} \Phi(z)\right| 0\right\rangle=\left\langle 0\left|O_{\mathcal{R}^{\prime}}^{1} \Phi(z) O_{\mathcal{R}^{\prime}}^{2}\right| 0\right\rangle,
$$

where we have used that the operator $O_{\mathcal{R}^{\prime}}^{2}$ is spacelike separated from the region where $\Phi$ is localized in order to move it to the right of $\Phi$. However, according to the Reeh-Schlieder theorem [46], any state $|\psi\rangle$ may be approximated to arbitrary precision by acting with a (smeared) set of local operators in any open set in spacetime. Since the region of points that are spacelike separated from a finite compact region is an open set, we may apply this idea to the right-hand side above to conclude that for any sates $\left|\psi_{i}\right\rangle$

$$
\left\langle\psi_{2}|\Phi| \psi_{1}\right\rangle=0
$$

This implies that $\Phi$ vanishes as an operator.

It is interesting to pinpoint exactly where this logic breaks down for non-local operators such as the average null energy operator $\mathcal{E}$. As long as the region that is spacelike separated to $\Phi$ is open, one may repeat the Reeh-Schlieder argument and prove that $\Phi$ vanishes even if it is non-local. The way the null energy operator $\mathcal{E}$ avoids this conclusion is that it has support along a complete null line and hence the region of points that are spacelike separated to $\mathcal{E}$ is not open, since it consists just of the codimension one null plane containing the null line. ${ }^{14}$

\footnotetext{
${ }^{14}$ We that H. Casini for an enlightening discussion on this point.
} 


\section{B Details of the collider calculation}

In this appendix we will give more details on the calculation of the energy expectation value for a conformal collider experiment that we considered in this paper, giving a bound on $C_{T T \mathcal{O}}$ in arbitrary dimensions.

\section{B.1 Normalized states}

The states that we consider for the collider experiment are superposition of states of normalized wavepackets. Following [1] we take the state defined as

$$
|\mathcal{O}(q, \lambda)\rangle \equiv \mathcal{N} \int d^{d} x e^{-i q x^{0}} \exp \left[-\frac{x_{0}^{2}+\vec{x}^{2}}{\sigma^{2}}\right] \lambda \cdot \mathcal{O}(x)|0\rangle, \quad q>0
$$

where $q \sigma \gg 1$. We find the normalization $\mathcal{N}$ by requiring the state to have unit norm in this limit. We will give their values only for the operators and polarizations relevant to computing the bound on $C_{T T \mathcal{O}}$. Namely for a scalar operator and for the stress tensor with polarization which is scalar with respect to the $\mathrm{SO}(d-2)$ symmetry perpendicular to $n$.

We normalize the scalar operator such that its two-point function is $\langle\mathcal{O}(x) \mathcal{O}(0)\rangle=$ $C_{\mathcal{O}} x^{-2 \Delta}$. Then the normalization condition for the state considered in the collider experiment is

$$
\langle\mathcal{O}(q) \mid \mathcal{O}(q)\rangle=1 \quad \Rightarrow \quad \mathcal{N}_{\mathcal{O}}^{-2}=C_{\mathcal{O}} \frac{2 \pi^{\frac{d+2}{2}}}{\Gamma(\Delta) \Gamma\left(\Delta-\frac{d}{2}+1\right)}\left(\frac{q}{2}\right)^{2 \Delta-d}
$$

where we used the following integral identity

$$
\int d^{d} x \frac{e^{i q x^{0}}}{x^{2 \Delta}}=\frac{2 \pi^{\frac{d+2}{2}}}{\Gamma(\Delta) \Gamma\left(\Delta-\frac{d}{2}+1\right)}\left(\frac{q}{2}\right)^{2 \Delta-d}, \quad q>0
$$

We will also need the proper normalization for the scalar state created by the stress tensor, which has the form

$$
\left|T\left(q, \lambda_{0}\right)\right\rangle \equiv \mathcal{N}_{T_{0}} \int d^{d} x e^{-i q x^{0}}\left(\lambda_{0}\right)_{i j} T_{i j}(x)|0\rangle,
$$

where we assumed the localized wavepacket limit. If we use conservation of the stress tensor we can chose the polarization along spatial directions. The normalized scalar polarization is

$$
\left(\lambda_{0}\right)_{i j}=\sqrt{\frac{d-1}{d-2}}\left[n_{i} n_{j}-\frac{1}{(d-1)} \delta_{i j}\right],
$$

which satisfies $\operatorname{Tr}\left(\lambda_{0}\right)=0$ and $\lambda_{0} \cdot \lambda_{0}=1$. In this case the normalization condition gives

$$
\left\langle T\left(q, \lambda_{0}\right) \mid T\left(q, \lambda_{0}\right)\right\rangle=1 \Rightarrow \mathcal{N}_{T_{0}}^{-1 / 2}=C_{T} \frac{4(d-1) \pi^{\frac{d}{2}+1}}{\Gamma\left(\frac{d}{2}\right) \Gamma(d+2)}\left(\frac{q}{2}\right)^{d},
$$

where the normalization of the two-point function is

$$
\left\langle T_{\mu \nu}(x) T_{\rho \sigma}(0)\right\rangle=\frac{C_{T}}{x^{2 d}} I_{\mu \nu \rho \sigma}(x),
$$


and the tensor structure that appears derived in [15] is

$$
\begin{aligned}
I_{\mu \nu \rho \sigma}(x) & =\frac{I_{\mu \rho}(x) I_{\nu \sigma}(x)+I_{\mu \sigma}(x) I_{\nu \rho}(x)}{2}-\frac{1}{d} g_{\mu \nu} g_{\rho \sigma}, \\
I_{\mu \nu}(x) & =g_{\mu \nu}-2 \frac{x_{\mu} x_{\nu}}{x^{2}} .
\end{aligned}
$$

Of course, by $\mathrm{SO}(d-1)$ rotational symmetry, the normalizations for $T_{1}$ and $T_{2}$ are also given by (B.6), once the polarizations are normalized to unity. Below we will perform the experiment of [1] for linear superpositions of these normalized states. But first we will review the form of the correlators we will need, mainly to fix notation and conventions.

\section{B.2 Three-point functions}

The three-point functions we will need are $\langle T T T\rangle,\langle T \mathcal{O O}\rangle$ and $\langle T T \mathcal{O}\rangle$. Their form were derived in [15] and the first two were studied in the context of the conformal collider in four dimensions in [1] and generalized to arbitrary dimensions in [3]. First, we will focus on $\langle T T \mathcal{O}\rangle$ which was not studied previously in the context of the conformal collider. The form consistent with conformal symmetry and conservation of the stress-tensor found in [15] is

$$
\left\langle T_{\mu \nu}\left(x_{1}\right) T_{\rho \sigma}\left(x_{2}\right) \mathcal{O}\left(x_{3}\right)\right\rangle=\frac{1}{x_{12}^{2 d-\Delta} x_{23}^{\Delta} x_{31}^{\Delta}} I_{\mu \nu} \mu^{\prime} \nu^{\prime}\left(x_{13}\right) I_{\rho \sigma^{\prime}}{ }^{\prime} \sigma^{\prime}\left(x_{23}\right) t_{\mu^{\prime} \nu^{\prime} \rho^{\prime} \sigma^{\prime}}\left(X_{12}\right),
$$

where $X_{12}=\frac{x_{13}}{x_{13}^{2}}-\frac{x_{23}}{x_{23}^{2}}$ and the tensor structure is a sum of three terms

$$
t_{\mu \nu \rho \sigma}(x) \equiv \hat{a} h_{\mu \nu \rho \sigma}^{1}(x)+\hat{b} h_{\mu \nu \rho \sigma}^{2}(x)+\hat{c} h_{\mu \nu \rho \sigma}^{3}(x),
$$

where each $h^{i}$ is traceless and symmetric under $\mu \nu \leftrightarrow \rho \sigma, x \rightarrow-x$

$$
\begin{aligned}
h_{\mu \nu \rho \sigma}^{1}(x) & =\frac{1}{x^{4}}\left(x_{\mu} x_{\nu} x_{\rho} x_{\sigma}+\ldots\right), \\
h_{\mu \nu \rho \sigma}^{2}(x) & =\frac{1}{x^{2}}\left(x_{\mu} x_{\rho} g_{\nu \sigma}+\ldots\right), \\
h_{\mu \nu \rho \sigma}^{3}(x) & =g_{\mu \rho} g_{\nu \sigma}+\ldots,
\end{aligned}
$$

where dots represent terms needed for expressions to be traceless and symmetric and in each line they involve a fixed number of factors of $x$. The main advantage of this approach is that it makes transparent the OPE limit $x_{2} \rightarrow x_{1}$, or equivalently taking the $x_{3} \rightarrow \infty$ limit

$$
T_{\mu \nu}(x) T_{\rho \sigma}(0) \sim \frac{1}{x^{2 d-\Delta}}\left(\hat{a} h_{\mu \nu \rho \sigma}^{1}(x)+\hat{b} h_{\mu \nu \rho \sigma}^{2}(x)+\hat{c} h_{\mu \nu \rho \sigma}^{3}(x)\right) \mathcal{O}(0) .
$$

Conservation can be imposed in this limit to the right hand side to the equation above, giving the two independent relations

$$
\begin{aligned}
\hat{a}+4 \hat{b}-\frac{1}{2}(d-\Delta)(d-1)(\hat{a}+4 \hat{b})-d \Delta \hat{b} & =0, \\
\hat{a}+4 \hat{b}+d(d-\Delta) \hat{b}+d(2 d-\Delta) \hat{c} & =0 .
\end{aligned}
$$

This fixes the three point function to a single conserved structure up to an overall coefficient, which we can define as

$$
C_{T T \mathcal{O}} \equiv \hat{a}+8(\hat{b}+\hat{c}) .
$$


Another standard way of representing conformal three-point functions is given by the spinning correlator formalism of [17]. We will write the correlator in terms of the embedding space coordinate $X_{i} \in \mathbb{R}^{d+1,1}$ and the polarization $Z_{i} \in \mathbb{R}^{d+1,1}$ such that $Z^{2}=0$. The correlator we need is in appendix A of [17] and in terms of the usual conformal structures $V_{i}$ and $H_{i j}$ is given by

$$
\left\langle T\left(X_{1}, Z_{1}\right) T\left(X_{2}, Z_{2}\right) \mathcal{O}\left(X_{3}\right)\right\rangle=\frac{\alpha_{1} V_{1}^{2} V_{2}^{2}+\alpha_{3} H_{12} V_{1} V_{2}+\alpha_{6} H_{12}^{2}}{\left(-2 X_{1} \cdot X_{2}\right)^{d+2-\frac{\Delta}{2}}\left(-2 X_{2} \cdot X_{3}\right)^{\frac{\Delta}{2}}\left(-2 X_{3} \cdot X_{1}\right)^{\frac{\Delta}{2}}},
$$

where $T(X, Z) \equiv Z^{A} Z^{B} T_{A B}(X)$ (with the index running from 0 to $d+1$ ) and the labels $1,3,6$ on the $\alpha$ 's correspond to the subset of the 10 structures that $\left\langle T T \mathcal{O}_{\ell}\right\rangle$ has for arbitrary $\ell$, that survive in the case $\ell=0$. The building blocks are

$$
\begin{aligned}
V_{1} & =\frac{\left(Z_{1} \cdot X_{2}\right)\left(X_{1} \cdot X_{3}\right)-\left(Z_{1} \cdot X_{3}\right)\left(X_{1} \cdot X_{2}\right)}{X_{2} \cdot X_{3}} \\
V_{2} & =\frac{\left(Z_{2} \cdot X_{3}\right)\left(X_{2} \cdot X_{1}\right)-\left(Z_{2} \cdot X_{1}\right)\left(X_{2} \cdot X_{3}\right)}{X_{1} \cdot X_{3}} \\
H_{12} & =-2\left(\left(Z_{1} \cdot Z_{2}\right)\left(X_{1} \cdot X_{2}\right)-\left(Z_{1} \cdot X_{2}\right)\left(Z_{2} \cdot X_{1}\right)\right) .
\end{aligned}
$$

Starting from the expression in $d+2$-dimensional embedding space we can obtain the $d$ dimensional correlator $\left\langle T\left(x_{1}, z_{1}\right) T\left(x_{2}, z_{2}\right) \mathcal{O}\left(x_{3}\right)\right\rangle$ by using the replacements $-2 X_{i} \cdot X_{j} \rightarrow$ $x_{i j}^{2}, Z_{i} \cdot Z_{j} \rightarrow z_{i} \cdot z_{j}$ and $X_{i} \cdot Z_{j} \rightarrow x_{i j} \cdot z_{j}$, where now in $d$-dimensions we define $T(x, z)=$ $z^{\mu} z^{\nu} T_{\mu \nu}(x)$, with the index running from 0 to $d-1$. Of course after these replacements the answer coincides with the Osborn-Petkou three-point function. We can match the coefficients of the different representations by taking the OPE limit. The result gives

$$
\begin{aligned}
& \alpha_{1}=\hat{a}+8(\hat{b}+\hat{c}), \\
& \alpha_{3}=4(\hat{b}+2 \hat{c}), \\
& \alpha_{6}=2 \hat{c},
\end{aligned}
$$

The OPE coefficient (B.18) is now $C_{T T \mathcal{O}}=\hat{a}+8(\hat{b}+\hat{c})=\alpha_{1}$. The conservation equations in terms of these parameters are

$$
\begin{aligned}
\alpha_{1}(2+\Delta-d(1-d+\Delta))+\alpha_{3}\left(-2-\Delta+\frac{d}{2}(\Delta+2)\right) & =0 \\
2 \alpha_{1}+\frac{1}{2} \alpha_{3}\left(-4+d^{2}-d \Delta\right)+\alpha_{6} d \Delta & =0 .
\end{aligned}
$$

which we can solve in terms of $C_{T T \mathcal{O}}$.

We defined in the main text the notation we will use for the stress-tensor three-point function. Another correlator we need is

$$
\left\langle T_{\mu \nu}\left(x_{1}\right) \mathcal{O}\left(x_{2}\right) \mathcal{O}\left(x_{3}\right)\right\rangle=C_{T \mathcal{O O}} \frac{1}{x_{12}^{d} x_{23}^{2 \Delta-d} x_{31}^{d}} I_{\mu \nu \rho \sigma}\left(x_{13}\right)\left(\frac{X_{12}^{\rho} X_{12}^{\sigma}}{X_{12}^{2}}-\frac{1}{d} g^{\rho \sigma}\right),
$$

which is fixed by a Ward identity to be $C_{T \mathcal{O O}}=-C_{\mathcal{O}} \frac{d \Delta}{(d-1) \Omega_{d-1}}$, with $\Omega_{d}$ being the are of a $S^{d}$ sphere. 


\section{B.3 Energy matrix}

As explained in the main text we want to consider states of the form

$$
|\Psi\rangle=v_{1}\left|T\left(q, \lambda_{0}\right)\right\rangle+v_{2}|\mathcal{O}(q)\rangle,
$$

where we take $v=\left(v_{1}, v_{2}\right) \in \mathbb{C}^{2}$ such that $\left|v_{1}\right|^{2}+\left|v_{2}\right|^{2}=1$. The energy one-point function in the collider experiment is

$$
\langle\Psi|\mathcal{E}(n)| \Psi\rangle=v^{\dagger}\left(\begin{array}{cc}
\left\langle T\left(q, \lambda_{0}\right)|\mathcal{E}(n)| T\left(q, \lambda_{0}\right)\right\rangle & \left\langle T\left(q, \lambda_{0}\right)|\mathcal{E}(n)| \mathcal{O}(q)\right\rangle \\
\left\langle T\left(q, \lambda_{0}\right)|\mathcal{E}(n)| \mathcal{O}(q)\right\rangle^{*} & \langle\mathcal{O}(q)|\mathcal{E}(n)| \mathcal{O}(q)\rangle
\end{array}\right) v
$$

In this section we will compute the entries of this matrix. The diagonal elements were already computed in $[1,3]$ and are given by

$$
\left\langle T\left(q, \lambda_{0}\right)|\mathcal{E}(n)| T\left(q, \lambda_{0}\right)\right\rangle=\frac{q}{\Omega_{d-2}}\left(1-\frac{d-3}{d-1} t_{2}-\frac{d(d-1)-4}{d^{2}-1} t_{4}\right),
$$

where

$$
t_{2}=\frac{\left(d^{2}-1\right)\left((d-3) N_{F}-2(d-2) N_{V}\right)}{(d-3)\left((d-1)\left(d N_{V}+N_{F}\right)+2 N_{B}\right)}, \quad t_{4}=\frac{\left(d^{2}-1\right)\left(N_{B}-N_{F}+N_{V}\right)}{2 N_{B}+(d-1)\left(d N_{V}+N_{F}\right)} .
$$

Using these expressions we can find the parameters we called $T_{0}, T_{1}$ and $T_{2}$ in the main text. They are given by equation (2.10) where the functions $\rho_{i}(d)$ are given by

$$
\begin{aligned}
& \rho_{0}(d)=\frac{1}{\Omega_{d-1}^{2}} \frac{d(d+1)(d-2)}{2(d-1)} \\
& \rho_{1}(d)=\frac{1}{\Omega_{d-1}^{2}} \frac{d(d+1)}{4} \\
& \rho_{2}(d)=\frac{1}{\Omega_{d-1}^{2}} \frac{d(d+1)(d-2)}{2(d-3)}
\end{aligned}
$$

The state created by a scalar operator gives

$$
\langle\mathcal{O}(q)|\mathcal{E}(n)| \mathcal{O}(q)\rangle=\frac{q}{\Omega_{d-2}} .
$$

Now we will obtain the off-diagonal element of this matrix we has not been computed in the literature. To perform the calculation in arbitrary dimensions it is convenient to use the spinning correlator formalism. Since we are computing an expectation value the correlator we need to consider is not time-ordered. The right $i \epsilon$ prescription for this purpose was explained in [1] and [3], and we will omit it here to ease the notation. We start from the $d$-dimensional expression

$$
\left\langle T\left(x_{1}, z_{1}\right) T\left(x_{2}, z_{2}\right) \mathcal{O}\left(x_{3}\right)\right\rangle=\frac{\alpha_{1} V_{1}^{2} V_{2}^{2}+\alpha_{3} H_{12} V_{1} V_{2}+\alpha_{6} H_{12}^{2}}{\left(x_{12}^{2}\right)^{d+2-\frac{\Delta}{2}}\left(x_{23}^{2}\right)^{\frac{\Delta}{2}}\left(x_{31}^{2}\right)^{\frac{\Delta}{2}}}
$$

We will chose $T\left(x_{2}, z_{2}\right)$ to be the insertion taken to infinity and giving $\mathcal{E}(n)$. First we take $z_{2}=m=(1, n)$ (we chose the mostly plus convention for the metric in Minkowski space). Therefore $T\left(x_{2}, z_{2}\right) \rightarrow \frac{1}{4} T_{--}\left(x_{2}\right)$. Then we take the limit

$$
x_{2} \cdot \bar{m} \rightarrow \infty, \quad \bar{m}=(-1, n) .
$$


To take this limit we can use the results of appendix F of [14], and obtain

$$
\lim _{x_{2} \cdot \bar{n} \rightarrow \infty}\left\langle T\left(x_{1}, z_{1}\right) r^{d-2} T_{--}\left(x_{2}\right) \mathcal{O}\left(x_{3}\right)\right\rangle=\frac{\alpha_{1} \hat{V}_{1}^{2} \hat{V}_{2}^{2}+\alpha_{3} \hat{H}_{12} \hat{V}_{1} \hat{V}_{2}+\alpha_{6} \hat{H}_{12}^{2}}{2^{d}\left(x_{12} \cdot m\right)^{d+2-\frac{\Delta}{2}}\left(x_{1}^{2}\right)^{\frac{\Delta}{2}}\left(x_{2} \cdot m\right)^{\frac{\Delta}{2}}}
$$

where the structures in this limit are

$$
\hat{V}_{1}=\frac{z_{1} \cdot m \frac{x_{1}^{2}}{2}-x_{1} \cdot z_{1} x_{12} \cdot m}{x_{2} \cdot m}, \quad \hat{V}_{2}=\frac{x_{1} \cdot m}{x_{1}^{2}}, \quad H_{12}=-z_{1} \cdot m
$$

We can get the correct polarization of the insertion $T\left(x_{1}, z_{1}\right)$ by replacing $z_{\mu} z_{\nu} \rightarrow \lambda_{\mu \nu}^{T}$, assuming $\lambda$ is already traceless and symmetric which is true for expression (B.5). To simplify the expressions we will choose $n=(1,0, \ldots, 0)$ and write the positions as $x=$ $\left(x^{+}, x^{-}, x^{\perp}\right)$, where $x^{+}=x \cdot \bar{m}=x^{1}+x^{0}, x^{-}=x \cdot m=x^{1}-x^{0}$ and $x^{\perp}$ corresponds to the $d-2$ transversal components. Then we can define

$$
\hat{G}=\lim _{x_{2}^{+} \rightarrow \infty}\left(x_{2}^{+} / 2\right)^{d-2}\left\langle\lambda_{0} \cdot T\left(x_{1}\right) T_{--}\left(x_{2}^{+}, x_{2}^{-}, 0\right) \mathcal{O}\left(x_{3}=0\right)\right\rangle
$$

which is given by

$$
\begin{aligned}
\hat{G}= & \alpha_{1} \lambda_{11}^{T} \frac{\left(x_{1}^{-}\right)^{2}\left(\frac{x_{1}^{4}}{4}-x_{1}^{1} x_{12}^{-} x_{1}^{2}+x_{1}^{1} x_{1}^{1}\left(x_{12}^{-}\right)^{2}-\frac{1}{d-2} \sum_{i_{\perp}}\left(x_{1}^{i_{\perp}}\right)^{2}\left(x_{12}^{-}\right)^{2}\right)}{2^{d}\left(x_{12}^{-}\right)^{d+2-\frac{\Delta}{2}}\left(x_{1}^{2}\right)^{\frac{\Delta}{2}+2}\left(x_{2}^{-}\right)^{\frac{\Delta}{2}+2}} \\
& +\alpha_{3} \lambda_{11}^{T} \frac{2^{-d}\left(x_{1}^{-}\right)\left(x_{1}^{1} x_{12}^{-}-\frac{1}{2} x_{1}^{2}\right)}{\left(x_{12}^{-}\right)^{d+2-\frac{\Delta}{2}}\left(x_{1}^{2}\right)^{\frac{\Delta}{2}+1}\left(x_{2}^{-}\right)^{\frac{\Delta}{2}+1}}+\alpha_{6} \lambda_{11}^{T} \frac{2^{-d}}{\left(x_{12}^{-}\right)^{d+2-\frac{\Delta}{2}}\left(x_{1}^{2}\right)^{\frac{\Delta}{2}}\left(x_{2}^{-}\right)^{\frac{\Delta}{2}}}
\end{aligned}
$$

First we do integral over $x_{2}^{-}$, using the following identity

$$
\int d x_{2}^{-} \frac{1}{\left(x_{2}^{-}-i \epsilon\right)^{b}\left(x_{12}^{-}-i \epsilon\right)^{a}}=\frac{2 \pi i}{\left(x_{1}^{-}-2 i \epsilon\right)^{a+b-1}} \frac{\Gamma(a+b-1)}{\Gamma(a) \Gamma(b)},
$$

where we made explicit the pole prescription. Finally, to take the limit of the localized wavepackets is equivalent to setting $x_{3} \rightarrow 0$ and make a Fourier transform with respect to $x_{1}$ with momentum $(q, 0, \ldots, 0)$, namely

$$
\int d^{d} x_{1} e^{-i q x_{1}^{0}} \int d x_{2}^{-} \hat{G}
$$

To do this we first integrate over the $d-2$ transverse directions $x_{1}^{\perp}$ and then integrate over the light-cone coordinates $x_{1}^{ \pm}$. Because of $\mathrm{SO}(d-2)$ invariance, the integrand only depends on $x_{1}^{+}, x_{1}^{-}$and $x_{1}^{\perp} \cdot x_{1}^{\perp}$. Then the integral can be written as

$$
\begin{aligned}
\int d^{d} x_{1} e^{-i q x_{1}^{0}} \int d x_{2}^{-} \hat{G}= & \frac{\Omega_{d-3}}{2} \int d x_{1}^{+} e^{-i \frac{q}{2} x_{1}^{+}} \int d x_{1}^{-} e^{i \frac{q}{2} x_{1}^{-}}, \\
& \times \int R^{d-3} d R F\left(x_{1}^{+}, x_{1}^{-},\left(x_{1}^{\perp}\right)^{2}=R^{2}\right),
\end{aligned}
$$


where we defined $F=\int d x_{2}^{-} \hat{G}$ to indicate the functional dependence explicitly. After performing these integrals we use conservation conditions to write $\alpha_{3}$ and $\alpha_{6}$ in terms of $\alpha_{1}=C_{T T \mathcal{O}}$ using equations (B.16) and (B.23). Combining the three structures gives

$$
\int d^{d} x_{1} e^{-i q x_{1}^{0}} \int d x_{2}^{-} \hat{G}=\frac{C_{T T \mathcal{O}} 2^{2-d}(d-1) \pi^{\frac{d}{2}+2} \Gamma(d+1)}{(d-2) \Gamma\left(\frac{\Delta}{2}+2\right)^{2} \Gamma\left(d-\frac{\Delta}{2}\right) \Gamma\left(\frac{d+\Delta}{2}\right)} \lambda_{0} \cdot n \cdot n\left(\frac{q}{2}\right)^{\Delta+1}
$$

In this expression we generalized the answer to arbitrary $n$ by replacing $\lambda_{11} \rightarrow \lambda_{i j} n^{i} n^{j}$. Finally, we need to replace the specific value of the polarization tensor (B.5) and the proper normalization of the collider states (B.6) and (B.2). The final answer for the off-diagonal entry of the energy matrix is

$$
\left\langle T\left(q, \lambda_{0}\right)|\mathcal{E}(n)| \mathcal{O}(q)\right\rangle=\frac{q}{\Omega_{d-2}} \frac{C_{T T \mathcal{O}}}{\sqrt{C_{T} C_{\mathcal{O}}}} h(\Delta),
$$

where

$$
h(\Delta) \equiv \frac{\pi^{\frac{d+1}{2}} \Gamma(d+1) \sqrt{\Gamma\left(\frac{d}{2}-1\right) \Gamma(d+2) \Gamma(\Delta) \Gamma\left(\Delta-\frac{d-2}{2}\right)}}{2^{d} \Gamma\left(\frac{d-1}{2}\right) \Gamma\left(\frac{\Delta}{2}+2\right)^{2} \Gamma\left(\frac{d+\Delta}{2}\right) \Gamma\left(d-\frac{\Delta}{2}\right)}
$$

Then the energy matrix that gives the expectation value for these superposition states is

$$
\begin{aligned}
\langle\Psi|\mathcal{E}(n)| \Psi\rangle & =v^{\dagger}\left(\begin{array}{cc}
T_{0} & \frac{C_{T T \mathcal{O}}}{\sqrt{C_{T} C_{\mathcal{O}}}} h(\Delta) \\
\frac{C_{T T \mathcal{O}}^{*}}{\sqrt{C_{T} C_{\mathcal{O}}}} h^{*}(\Delta) & 1
\end{array}\right) v \\
T_{0} & \equiv 1-\frac{d-3}{d-1} t_{2}-\frac{d(d-1)-4}{d^{2}-1} t_{4}
\end{aligned}
$$

Having computed the energy matrix the next step is to impose ANEC, which is equivalent to imposing positivity of the energy expectation value for the collider experiment. This means that for all states

$$
|\Psi(v)\rangle=v_{1}\left|T\left(q, \lambda_{0}\right)\right\rangle+v_{2}|\mathcal{O}(q)\rangle
$$

we need to impose

$$
\langle\Psi(v)|\mathcal{E}(n)| \Psi(v)\rangle>0, \quad \forall v \in \mathbb{C}^{2} .
$$

This constraint is equivalent to the positivity of all the leading principal minors of the energy matrix. The first constraint is

$$
T_{0}=1-\frac{d-3}{d-1} t_{2}-\frac{d(d-1)-4}{d^{2}-1} t_{4}=\rho_{0}(d)\left(\frac{N_{B}}{C_{T}}\right) \geq 0
$$

which is the same as the original constraints of [1] and [3]. The next minor imposes the positivity of the $2 \times 2$ matrix which is

$$
\frac{\left|C_{T T \mathcal{O}}\right|^{2}}{C_{T} C_{\mathcal{O}}}|h(\Delta)|^{2} \leq T_{0}
$$

We can write $T_{0}$ and $C_{T}$ in terms of the $\langle T T T\rangle$ structures $N_{B}, N_{F}$ and $N_{V}$. This gives the equivalent expression that we quoted in the introduction

$$
\frac{\left|C_{T T \mathcal{O}}\right|^{2}}{C_{\mathcal{O}}} f(\Delta) \leq N_{B}
$$


where

$$
f(\Delta)=\frac{(d-1)^{3} d \pi^{2 d} \Gamma\left(\frac{d}{2}\right) \Gamma(d+1) \Gamma(\Delta) \Gamma\left(\Delta-\frac{d-2}{2}\right)}{(d-2)^{2} \Gamma\left(\frac{\Delta}{2}+2\right)^{4} \Gamma\left(\frac{d+\Delta}{2}\right)^{2} \Gamma\left(d-\frac{\Delta}{2}\right)^{2}}
$$

These two conditions (B.53) and (B.54) are necessary and sufficient for the energy to be positive for any state of the form (B.51). For operators $\mathcal{O}$ that are not hermitian this bound does not have information about the phase of the OPE coefficient $C_{T T \mathcal{O}}$.

\section{Free scalar correlators}

In this section we will present some details on the calculation of the TTO correlators for a free scalar that saturates the bound above. We use the normalization of [15] for

$$
\langle\phi(x) \phi(0)\rangle=\frac{1}{(d-2) \Omega_{d-1}} \frac{1}{x^{d-2}} .
$$

and the stress tensor is defined as

$$
T_{\mu \nu}=: \partial_{\mu} \phi \partial_{\nu} \phi:-\frac{1}{4(d-1)}\left((d-2) \partial_{\mu} \partial_{\nu}+g_{\mu \nu} \partial^{2}\right): \phi^{2}: .
$$

Since scalar operators have integer dimensions we only need to consider $\mathcal{O}$ such that $\Delta<2 d$. The first one is $\mathcal{O} \sim \phi$. This one is predicted to vanish since $f\left(\Delta=\frac{d-2}{2}\right) \rightarrow \infty$. This is indeed the case since an odd number of fields appear in $\langle T T \phi\rangle$. The next operator is $\mathcal{O}=: \phi^{2}$ : of dimension $\Delta=d-2$. The correct normalization of the two-point function gives

$$
\left\langle: \phi^{2}:(x): \phi^{2}:(0)\right\rangle=\frac{2}{(d-2)^{2} \Omega_{d-1}^{2}} \frac{1}{x^{2(d-2)}}, \quad C_{\mathcal{O}}=\frac{2}{(d-2)^{2} \Omega_{d-1}^{2}} .
$$

Using Wick contractions we can also compute $\left\langle T T \phi^{2}\right\rangle$. One can check that the answer has the conformal invariant structure (B.19) with

$$
C_{T T \mathcal{O}}=\alpha_{1}=\frac{(d-2) d^{2}}{2(d-1)^{2}} \frac{1}{\Omega_{d-1}^{3}}, \quad \alpha_{3}=-\frac{4}{d-2} \alpha_{1}, \quad \alpha_{6}=\frac{2}{(d-2) d} \alpha_{1}
$$

Finally, the function appearing in the bound takes the value

$$
f(\Delta=d-2)=\frac{8(d-1)^{4} \pi^{2 d}}{(d-2)^{4} \Gamma\left(\frac{d}{2}+1\right)^{4}}
$$

Putting everything together we find that the bound is saturated

$$
\frac{\left|C_{T T \mathcal{O}}\right|^{2}}{C_{\mathcal{O}}} f(d-2)=1 \leq N_{B}=1 \text {. }
$$

We have seen that the bound is saturated by a scalar field with $\mathcal{O}=: \phi^{2}:$. Nevertheless there is one more primary scalar field we can make of dimension less than $2 d$, namely $\mathcal{O}=: \phi^{4}$ : which has dimension $\Delta_{\phi^{4}}=2(d-2)$. Working out the Wick contractions we can verify that $\left\langle T T: \phi^{4}:\right\rangle=0$. We can argue more generally that this is so. The form of the correlator (B.19) indicates that there is a $x_{1} \rightarrow x_{2}$ singularity whenever $\Delta<2 d$. On the other hand, to have a non-zero answer we can only take a Wick contraction which is between $T$ and $: \phi^{4}$ : but not between the stress tensors. Therefore if this calculation would give a non-zero answer, it will be finite when $x_{1} \rightarrow x_{2}$. The only way this is consistent with the form of the correlator fixed by conformal symmetry (B.19) is if it indeed vanishes. 


\section{$\mathrm{D}\langle T T O\rangle$ parity-odd structures in $d=3$}

In dimensions $d \geq 4$ the three-point function $\langle T T O\rangle$ has only a parity-even structure consistent with permutation symmetry and conservation of the stress-tensors. The situation for $d=3$ is special since only for this number of dimensions a new parity-odd structure appears, that is also consistent with all the requirements. In this case the full correlator is

$$
\langle T T \mathcal{O}\rangle=\langle T T \mathcal{O}\rangle_{\text {even }}+\langle T T \mathcal{O}\rangle_{\text {odd }}
$$

where the parity-even part coincides with the answer for $d \geq 4$

$$
\left\langle T\left(X_{1}, Z_{1}\right) T\left(X_{2}, Z_{2}\right) \mathcal{O}\left(X_{3}\right)\right\rangle_{\text {even }}=\frac{\alpha_{1} V_{1}^{2} V_{2}^{2}+\alpha_{3} H_{12} V_{1} V_{2}+\alpha_{6} H_{12}^{2}}{X_{12}^{5-\frac{\Delta}{2}} X_{23}^{\frac{\Delta}{2}} X_{31}^{\frac{\Delta}{2}}},
$$

and the new structure is

$$
\left\langle T\left(X_{1}, Z_{1}\right) T\left(X_{2}, Z_{2}\right) \mathcal{O}\left(X_{3}\right)\right\rangle_{\text {odd }}=\frac{\beta_{1} V_{1} V_{2}+\beta_{2} H_{12}}{X_{12}^{5-\frac{\Delta}{2}} X_{23}^{\frac{\Delta}{2}} X_{31}^{\frac{\Delta}{2}}} \epsilon\left(Z_{1}, Z_{2}, X_{1}, X_{2}, X_{3}\right),
$$

Since conservation put constraints independently for $\alpha_{1,3,6}$ and $\beta_{1,2}$ we can forget about the parity-even part and we get $\beta_{1}(\Delta-3)-\beta_{2}(\Delta+1)=0$. Therefore the parity-odd structure is also fixed by a single OPE coefficient which we denote $C_{T T O}^{\text {odd }}$, as opposed to the one in the even part $C_{T T O}^{\text {even }}=\alpha_{1}$. For completeness we present the same correlator in the Osborn and Petkou formalism

$$
\left\langle T_{\mu \nu}\left(x_{1}\right) T_{\rho \sigma}\left(x_{2}\right) \mathcal{O}\left(x_{3}\right)\right\rangle=\frac{1}{x_{12}^{6-\Delta} x_{23}^{\Delta} x_{31}^{\Delta}} I_{\mu \nu}^{\mu^{\prime} \nu^{\prime}}\left(x_{13}\right) I_{\rho \sigma} \rho^{\rho^{\prime} \sigma^{\prime}}\left(x_{23}\right) t_{\mu^{\prime} \nu^{\prime} \rho^{\prime} \sigma^{\prime}}\left(X_{12}\right),
$$

where $t=\hat{d} t^{1}+\hat{e} t^{2}$ and we define

$$
\begin{aligned}
t_{\mu \nu \rho \sigma}^{1}(X) & =\frac{x_{\mu} x_{\rho} x_{\gamma}}{x^{3}} \varepsilon_{\nu \sigma \gamma}+\ldots, \\
t_{\mu \nu \rho \sigma}^{2}(X) & =\frac{\delta_{\mu \rho} x_{\gamma}}{x} \varepsilon_{\nu \sigma \gamma}+\ldots,
\end{aligned}
$$

where the dots represent other terms of the same form to make the answer symmetric, traceless and permutation symmetric. Conservation imposes $\hat{e}(\Delta-7)+\hat{d}(\Delta-3)=0$. Either in the Osborn and Petkou formalism or in the spinning correlator formalism, we define the parity-odd OPE coefficient as $C_{T T O}^{\text {odd }} \equiv \hat{d}+\hat{e}=\left(\beta_{2}-\beta_{1}\right) / 4$.

\section{E $\langle T T J\rangle$ three-point function}

In this appendix we will provide some details on the CFT three-point function controlling the mixed gauge-gravitational anomaly $\langle T T J\rangle$. Imposing permutation symmetry between the stress-tensors and conservation, this correlator only involves an allowed parity-odd structure. In the spinning correlator formalism it is given by

$$
\left\langle T\left(X_{1}, Z_{1}\right) T\left(X_{2}, Z_{2}\right) J\left(X_{3}, Z_{3}\right)\right\rangle \sim \frac{H_{12}-4 V_{1} V_{2}}{X_{12}^{4} X_{23}^{2} X_{31}^{2}} \epsilon\left(Z_{1}, Z_{2}, Z_{3}, X_{1}, X_{2}, X_{3}\right),
$$


where as usual the upper-case denote coordinates in embedding space and $H_{i j}$ and $V_{i}$ are the usual structures defined in [17]. From this expression it is possible to deduce the conservation equation for the current when the CFT is placed on a curved background and gives the right normalization for $C_{T T J}$ [24]. Then the three-point function is

$$
\left\langle T\left(X_{1}, Z_{1}\right) T\left(X_{2}, Z_{2}\right) J\left(X_{3}, Z_{3}\right)\right\rangle=\frac{C_{T T J}}{2 \pi^{6}} \frac{H_{12}-4 V_{1} V_{2}}{X_{12}^{4} X_{23}^{2} X_{31}^{2}} \epsilon\left(Z_{1}, Z_{2}, Z_{3}, X_{1}, X_{2}, X_{3}\right)
$$

For completeness we can write this same correlator using the notation of Osborn and Petkou. This can be written as

$$
\left\langle T_{\mu \nu}\left(x_{1}\right) T_{\rho \sigma}\left(x_{2}\right) J_{\alpha}\left(x_{3}\right)\right\rangle=\frac{1}{x_{12}^{5} x_{23}^{3} x_{31}^{3}} I_{\mu \nu}^{\mu^{\prime} \nu^{\prime}}\left(x_{13}\right) I_{\rho \sigma} \rho^{\prime} \sigma^{\prime}\left(x_{23}\right) t_{\mu^{\prime} \nu^{\prime} \rho^{\prime} \sigma^{\prime} \alpha}\left(X_{12}\right)
$$

where $t_{\mu \nu \rho \sigma \alpha}(x)$ is the OPE structure $T_{\mu \nu}(x) T_{\rho \sigma}(0) \sim|x|^{-5} t_{\mu \nu \rho \sigma \alpha} J^{\alpha}(0)$ and $X_{12}=\frac{x_{13}}{x_{13}^{2}}-$ $\frac{x_{23}}{x_{23}}$. The two structures possible, which are linear combinations of the $H_{12}$ and $V_{1} V_{2}$, are explicitly

$$
t_{\mu \nu \rho \sigma \alpha}^{1}(x)=\frac{x_{\gamma} x_{\mu} x_{\rho}}{4|x|^{3}} \epsilon_{\nu \sigma \alpha \gamma}+\ldots
$$

and

$$
t_{\mu \nu \rho \sigma \alpha}^{2}(x)=\frac{x_{\gamma} \delta_{\mu \rho}}{4|x|} \epsilon_{\nu \sigma \alpha \gamma}+\ldots,
$$

where the dots represent terms needed to add in ordered for the expression to be symmetric, traceless and permutation symmetric between the first two pair of indices. The most general case has $t=\hat{a} t^{1}+\hat{b} t^{2}$. Imposing conservation and comparing with the spinning correlator formalism we get $\hat{a}=-6 \hat{b}=3 C_{T T J} / \pi^{6}$. Using this information it is straightforward to apply the same procedure as was done for $\langle T T O\rangle$ to obtain the energy matrix elements in the conformal collider experiment.

\section{F Computing the bound in the gravity theory}

In this appendix we relate the OPE coefficient $C_{T T \mathcal{O}}$ to a coefficient, $\alpha$, in the $A d S_{D}$ effective action

$$
S=\frac{M_{p l}^{D-2}}{2}\left[\int \sqrt{g}(R-2 \Lambda)+(\nabla \chi)^{2}-m^{2} \chi^{2}+2 \alpha \chi W^{2}\right], \quad \Lambda=-\frac{(D-1)(D-2)}{2 R_{\text {AdS }}^{2}},
$$

where $D$ is the dimension of $A d S_{D} \cdot \chi$ is defined to be dimensionless and $\alpha$ has dimensions of length squared.

In principle we can compute the relation between $\alpha$ and $C_{T T \mathcal{O}}$ by computing the three point function between a scalar and the graviton produced by this cubic term in the Lagrangian, using Witten diagrams. Instead, we will follow a different route. We will directly compute the energy correlator in gravity and derive a bound on $\alpha$ by demanding its positivity. We then relate $\alpha$ and $C_{T T \mathcal{O}}$ by demanding that this gravity bound, in terms of $\alpha$, matches the bound we obtained in terms of $C_{T T \mathcal{O}}$ in the field theory analysis.

We will rely on $[1,3]$ where the energy correlators were computed in gravity. An important point is that the insertion of $T_{--}$corresponds to a shock wave localized in a null 
plane. Furthermore, an operator insertion at the origin with definite energy-momentum gives rise to an excitation that crosses this null plane at a localized point. For this reason the computation of the bound boils down to analyzing the propagation of an excitation through a suitable gravitational shock wave in flat space. The $A d S_{D}$ space is only relevant for determining the transverse profile of the shock wave, as we will see below.

For these reasons we consider a shock wave of the form

$$
d s^{2}=d s_{\text {flat }}^{2}+\left(d x^{+}\right)^{2} \delta\left(x^{+}\right) h(y), \quad d s_{\text {flat }}^{2}=-d x^{+} d x^{-}+d y^{2} .
$$

Adding gravitons we get

$$
d s^{2}=d s_{\text {flat }}^{2}+\left(d x^{+}\right)^{2} \delta\left(x^{+}\right) h(y)+d x^{\mu} d x^{\nu} \zeta_{\mu} \zeta_{\nu} e^{i p \cdot x} G(p)+d x^{\mu} d x^{\nu} \bar{\zeta}_{\mu} \bar{\zeta}_{\nu} e^{-i p \cdot x} \bar{G}(p),
$$

with $\zeta^{2}=0, \zeta^{\mu} p_{\mu}=0$. Note that the graviton polarization is $\zeta_{\mu \nu}=\zeta_{\mu} \zeta_{\nu}$, and is normalized to one $\zeta \cdot \bar{\zeta}=1$. We can think of $G(p)$ and $\bar{G}(p)$ as complex numbers, which in the quantum theory will be related to $a$ and $a^{\dagger}$. Inserting (F.3) into (F.1) we can derive the quadratic and cubic interaction terms.

$$
\begin{gathered}
S=\frac{M_{p l}^{D-2}}{2} \int d x^{+} d x^{-} d^{D-2} y\{[ \\
{\left[p_{+} p_{-}+\delta\left(x^{+}\right) p_{-}^{2} h\right]\left[G(p) \bar{G}(p)+4 p_{-} p_{+} \chi(p) \bar{\chi}(p)\right]+} \\
\left.+8 p_{-}^{2} \alpha \zeta^{i j} \partial_{i} \partial_{j} h \delta\left(x^{+}\right) G(p) \bar{\chi}(p)+c . c .\right\},
\end{gathered}
$$

where we only wrote the terms relevant for our computation, ignoring transverse derivatives in the kinetic terms. Momentarily setting the scalar field to zero, we see that we have the following equation for the graviton as it crosses the shock wave

$$
\left.\Delta h_{\mu \nu} \equiv h_{\mu \nu}\right|_{x^{+}=0^{+}}-\left.h_{\mu \nu}\right|_{x^{+}=0^{-}}=i p_{-} h h_{\mu \nu} .
$$

Exponentiating this, $h_{\mu \nu}\left(x^{+}=0^{+}\right)=e^{i p_{-} h} h_{\mu \nu}\left(x^{+}=0^{-}\right)$, we see that the time delay is simply given by $h$. This is as expected from (F.2) since we can shift $x^{-}$by $h$ and make the term involving $h$ disappear if we ignore its $y$ dependence. So far, we considered the computation in flat space. An insertion of the null energy integrated along a ray in the boundary theory gives rise to a shockwave in $A d S_{D}$ which is localized on a null direction. Its dependence on the transverse directions is the following. The transverse space is an $H_{D-2}$. This is easy to see in embedding coordinates where $A d S_{D}$ is $\tilde{W}^{+} \tilde{W}^{-}+W^{\mu} W_{\nu}=-1$ (setting $R_{A d S_{D}}=1$ ). The null plane is $\tilde{W}^{+}=0$. It contains the null direction parametrized by $\tilde{W}^{-}$as well as the transverse space $W^{\mu} W_{\mu}=-1$. The profile of the wave is proportional to $h \propto\left(W^{0}-W^{i} n^{i}\right)^{2-D}[1,3]$, with a positive coefficient. Here $\vec{n}^{i}$ is a vector on the sphere at infinity in the boundary Minkowski space. For (F.4) we need the derivatives at $W^{i}=0$, which are given by

$$
h \rightarrow h, \quad \partial_{i} \partial_{j} h=\left[(\text { constant }) \delta_{i j}+(D-2)(D-1) n_{i} n_{j}\right] h \frac{1}{R_{A d S_{D}}^{2}},
$$

where the constant does not matter because the graviton is traceless. The relevant component of the graviton is the one with polarization along $n^{i}$. This has the expression

$$
\zeta_{i j}=\sqrt{\frac{D-2}{D-3}}\left[n^{i} n^{j}-\frac{\delta_{i j}}{D-2}\right] .
$$


The expression for the time delay acting on a superposition of a graviton and a scalar is now a matrix proportional to

$$
\left(\begin{array}{ll}
1 & \gamma \\
\gamma & 1
\end{array}\right), \quad \gamma \equiv 4(D-1) \sqrt{(D-3)(D-2)} \frac{\alpha}{R_{A d S_{D}}^{2}},
$$

where the matrix is acting on a two dimensional space where one direction is the scalar and the other is the graviton with polarization (F.7). The unitarity bound comes from the restriction that the eigenvalues are non-negative, or $|\gamma| \leq 1$, which is

$$
\frac{|\alpha|}{R_{A d S_{D}}^{2}} \leq \frac{1}{4(D-1) \sqrt{(D-3)(D-2)}}=\frac{1}{4 d \sqrt{(d-2)(d-1)}},
$$

where $d$ is the dimension of the boundary. Comparing this with the bound obtained in (3.8), with the non-Einstein-gravity structures set to zero, we obtain (6.2). Of course, once we get the proportionality constant between $\alpha$ and $C_{T T \mathcal{O}}$ for the Einstein gravity case, the same constant holds also if we add the purely gravitational higher derivative terms that generate the other tensor structures for $\langle T T T\rangle$. We could add them to this computation, but we expect to reproduce the bounds we got in the general field theory analysis.

\section{F.1 Four-dimensional case}

In the special case of the four dimensional theory, we actually have two couplings (6.3). This leads to a new interaction term in (F.4) of the form

$$
\alpha \zeta^{i j} \partial_{i} \partial_{j} h \rightarrow \alpha_{e} \zeta^{i j} \partial_{i} \partial_{j} h+\alpha_{o} \zeta^{i l} \epsilon_{l j} \partial_{i} \partial_{j} h
$$

where now $\epsilon_{i j}$ is the two dimensional epsilon symbol. This means that the scalar can now mix with the other graviton polarization component besides (F.7). Namely, defining (F.7) as $\zeta_{\oplus}$, it can also mix with $\zeta_{\otimes}^{i j} \equiv \epsilon^{i l} \zeta_{\oplus}^{l j}$. Now the time delay is a three by three matrix

$$
\left(\begin{array}{ccc}
1 & \gamma & \beta \\
\gamma & 1 & 0 \\
\beta & 0 & 1
\end{array}\right), \quad \gamma \equiv 12 \sqrt{2} \frac{\alpha_{e}}{R_{A d S_{4}}^{2}}, \quad \beta \equiv 12 \sqrt{2} \frac{\alpha_{o}}{R_{A d S_{4}}^{2}},
$$

where the rows and columns correspond to the scalar and the two graviton polarizations. Now the bound is (6.5). Comparing this to (4.13), after setting the non-Einstein-gravity structures to zero, we get the precise mapping to the $C_{T T \mathcal{O}}$ coefficients (6.4).

Open Access. This article is distributed under the terms of the Creative Commons Attribution License (CC-BY 4.0), which permits any use, distribution and reproduction in any medium, provided the original author(s) and source are credited.

\section{References}

[1] D.M. Hofman and J. Maldacena, Conformal collider physics: energy and charge correlations, JHEP 05 (2008) 012 [arXiv:0803.1467] [INSPIRE]. 
[2] J. de Boer, M. Kulaxizi and A. Parnachev, $A d S_{7} / C F T_{6}$, Gauss-Bonnet gravity and viscosity bound, JHEP 03 (2010) 087 [arXiv: 0910.5347] [INSPIRE].

[3] A. Buchel, J. Escobedo, R.C. Myers, M.F. Paulos, A. Sinha and M. Smolkin, Holographic GB gravity in arbitrary dimensions, JHEP 03 (2010) 111 [arXiv:0911.4257] [INSPIRE].

[4] T. Faulkner, R.G. Leigh, O. Parrikar and H. Wang, Modular Hamiltonians for deformed half-spaces and the averaged null energy condition, JHEP 09 (2016) 038 [arXiv:1605.08072] [INSPIRE].

[5] T. Hartman, S. Kundu and A. Tajdini, Averaged null energy condition from causality, JHEP 07 (2017) 066 [arXiv: 1610.05308] [INSPIRE].

[6] T. Hartman, S. Jain and S. Kundu, A new spin on causality constraints, JHEP 10 (2016) 141 [arXiv: 1601.07904] [INSPIRE].

[7] D.M. Hofman, D. Li, D. Meltzer, D. Poland and F. Rejon-Barrera, A proof of the conformal collider bounds, JHEP 06 (2016) 111 [arXiv:1603.03771] [INSPIRE].

[8] S.D. Chowdhury, J.R. David and S. Prakash, Constraints on parity violating conformal field theories in $d=3$, arXiv: 1707.03007 [INSPIRE].

[9] A. Dymarsky, F. Kos, P. Kravchuk, D. Poland and D. Simmons-Duffin, The 3d stress-tensor bootstrap, arXiv:1708.05718 [INSPIRE].

[10] R.W. Fuller and J.A. Wheeler, Causality and multiply connected space-time, Phys. Rev. 128 (1962) 919 [INSPIRE].

[11] H. Epstein, V. Glaser and A. Jaffe, Nonpositivity of energy density in quantized field theories, Nuovo Cim. 36 (1965) 1016 [inSPIRE].

[12] J.L. Friedman, K. Schleich and D.M. Witt, Topological censorship, Phys. Rev. Lett. 71 (1993) 1486 [Erratum ibid. 75 (1995) 1872] [gr-qc/9305017] [INSPIRE].

[13] S. Balakrishnan, T. Faulkner, Z.U. Khandker and H. Wang, A general proof of the quantum null energy condition, arXiv:1706.09432 [INSPIRE].

[14] Z. Komargodski, M. Kulaxizi, A. Parnachev and A. Zhiboedov, Conformal field theories and deep inelastic scattering, Phys. Rev. D 95 (2017) 065011 [arXiv: 1601.05453] [INSPIRE].

[15] H. Osborn and A.C. Petkou, Implications of conformal invariance in field theories for general dimensions, Annals Phys. 231 (1994) 311 [hep-th/9307010] [INSPIRE].

[16] A. Zhiboedov, On conformal field theories with extremal a/c values, JHEP 04 (2014) 038 [arXiv: 1304.6075] [INSPIRE].

[17] M.S. Costa, J. Penedones, D. Poland and S. Rychkov, Spinning conformal correlators, JHEP 11 (2011) 071 [arXiv:1107.3554] [INSPIRE].

[18] M. Gillioz, X. Lu and M.A. Luty, Scale anomalies, states and rates in conformal field theory, JHEP 04 (2017) 171 [arXiv: 1612.07800] [INSPIRE].

[19] D. Pappadopulo, S. Rychkov, J. Espin and R. Rattazzi, OPE convergence in conformal field theory, Phys. Rev. D 86 (2012) 105043 [arXiv: 1208.6449] [inSPIRE].

[20] S. Giombi, S. Prakash and X. Yin, A note on CFT correlators in three dimensions, JHEP 07 (2013) 105 [arXiv:1104.4317] [INSPIRE].

[21] J. Maldacena and A. Zhiboedov, Constraining conformal field theories with a slightly broken higher spin symmetry, Class. Quant. Grav. 30 (2013) 104003 [arXiv:1204.3882] [INSPIRE]. 
[22] E. Sezgin, E.D. Skvortsov and Y. Zhu, Chern-Simons matter theories and higher spin gravity, JHEP 07 (2017) 133 [arXiv:1705.03197] [INSPIRE].

[23] S. El-Showk, M.F. Paulos, D. Poland, S. Rychkov, D. Simmons-Duffin and A. Vichi, Solving the $3 d$ Ising model with the conformal bootstrap II. c-minimization and precise critical exponents, J. Stat. Phys. 157 (2014) 869 [arXiv:1403.4545] [InSPIRE].

[24] J. Erdmenger, Gravitational axial anomaly for four-dimensional conformal field theories, Nucl. Phys. B 562 (1999) 315 [hep-th/9905176] [inSPIRE].

[25] R. Delbourgo and A. Salam, The gravitational correction to PCAC, Phys. Lett. B 40 (1972) 381 [INSPIRE].

[26] T. Eguchi and P.G.O. Freund, Quantum gravity and world topology, Phys. Rev. Lett. 37 (1976) 1251 [inSPIRE].

[27] L. Álvarez-Gaumé and E. Witten, Gravitational anomalies, Nucl. Phys. B 234 (1984) 269 [INSPIRE].

[28] H. Osborn, $N=1$ superconformal symmetry in four-dimensional quantum field theory, Annals Phys. 272 (1999) 243 [hep-th/9808041] [INSPIRE].

[29] D.T. Son and P. Surowka, Hydrodynamics with triangle anomalies, Phys. Rev. Lett. 103 (2009) 191601 [arXiv:0906.5044] [INSPIRE].

[30] A. Vilenkin, Parity nonconservation and rotating black holes, Phys. Rev. Lett. 41 (1978) 1575 [INSPIRE].

[31] K. Landsteiner, E. Megias and F. Pena-Benitez, Gravitational anomaly and transport, Phys. Rev. Lett. 107 (2011) 021601 [arXiv:1103.5006] [INSPIRE].

[32] K. Landsteiner, E. Lopez and G. Milans del Bosch, Quenching the CME via the gravitational anomaly and holography, arXiv:1709.08384 [INSPIRE].

[33] J. Gooth et al., Experimental signatures of the mixed axial-gravitational anomaly in the Weyl semimetal NbP, Nature 547 (2017) 324 [arXiv:1703.10682] [INSPIRE].

[34] X.O. Camanho, J.D. Edelstein, J. Maldacena and A. Zhiboedov, Causality constraints on corrections to the graviton three-point coupling, JHEP 02 (2016) 020 [arXiv:1407.5597] [INSPIRE].

[35] A. Bhattacharyya, L. Cheng and L.-Y. Hung, Relative entropy, mixed gauge-gravitational anomaly and causality, JHEP 07 (2016) 121 [arXiv: 1605.02553] [INSPIRE].

[36] J.M. Maldacena and G.L. Pimentel, On graviton non-Gaussianities during inflation, JHEP 09 (2011) 045 [arXiv:1104.2846] [INSPIRE].

[37] A. Lue, L.-M. Wang and M. Kamionkowski, Cosmological signature of new parity violating interactions, Phys. Rev. Lett. 83 (1999) 1506 [astro-ph/9812088] [INSPIRE].

[38] S. Alexander and J. Martin, Birefringent gravitational waves and the consistency check of inflation, Phys. Rev. D 71 (2005) 063526 [hep-th/0410230] [INSPIRE].

[39] N. Arkani-Hamed and J. Maldacena, Cosmological collider physics, arXiv:1503.08043 [INSPIRE].

[40] S. Saito, K. Ichiki and A. Taruya, Probing polarization states of primordial gravitational waves with CMB anisotropies, JCAP 09 (2007) 002 [arXiv:0705.3701] [INSPIRE]. 
[41] D. Baumann, H. Lee and G.L. Pimentel, High-scale inflation and the tensor tilt, JHEP 01 (2016) 101 [arXiv: 1507.07250] [INSPIRE].

[42] J.M. Maldacena, Non-Gaussian features of primordial fluctuations in single field inflationary models, JHEP 05 (2003) 013 [astro-ph/0210603] [INSPIRE].

[43] N. Bartolo and G. Orlando, Parity breaking signatures from a Chern-Simons coupling during inflation: the case of non-Gaussian gravitational waves, JCAP 07 (2017) 034 [arXiv: 1706.04627] [INSPIRE].

[44] J. Soda, H. Kodama and M. Nozawa, Parity violation in graviton non-Gaussianity, JHEP 08 (2011) 067 [arXiv:1106.3228] [INSPIRE].

[45] P. Creminelli and M. Zaldarriaga, Single field consistency relation for the 3-point function, JCAP 10 (2004) 006 [astro-ph/0407059] [INSPIRE].

[46] H. Reeh and S. Schlieder, Bemerkungen zur Unitäräquivalenz von Lorentzinvarianten Felden (in German), Nuovo Cim. 22 (1961) 1051. 